\title{
GlobalFungi: Global database of fungal records from high-throughput-sequencing metabarcoding studies
}

Tomáš Větrovský ${ }^{1}$, Daniel Morais ${ }^{1}$, Petr Kohout ${ }^{1}$, Clémentine Lepinay ${ }^{1}$, Camelia Algora Gallardo ${ }^{1}$, Sandra Awokunle Hollá ${ }^{1}$, Barbara Doreen Bahnmann ${ }^{1}$, Květa Bílohnědá ${ }^{1}$, Vendula Brabcová ${ }^{1}$, Federica D'Alòㄹ Zander Rainier Human ${ }^{1}$, Mayuko Jomura ${ }^{3}$, Miroslav Kolařík ${ }^{1}$, Jana Kvasničková ${ }^{1}$, Salvador Lladó ${ }^{1}$, Rubén López-Mondéjar ${ }^{1}$, Tijana Martinović ${ }^{1}$, Tereza Mašínová ${ }^{1}$, Lenka Meszárošová ${ }^{1}$, Lenka Michalčíková ${ }^{1}$, Tereza Michalová ${ }^{1}$, Sunil Mundra ${ }^{4}$, Diana Navrátilová ${ }^{1}$, Iñaki Odriozola ${ }^{1}$, Sarah Piché-Choquette ${ }^{1}$, Martina Štursová ${ }^{1}$, Karel Švec ${ }^{1}$, Vojtěch Tláskal ${ }^{1}$, Michaela Urbanová ${ }^{1}$, Lukáš Vlk ${ }^{1}$, Jana Vořišková ${ }^{1}$, Lucia Žifčákováa ${ }^{1}$, Petr Baldrian $^{1}$

\section{Affiliations}

1. Institute of Microbiology of the Czech Academy of Sciences, Vídeňská 1083, 14220 Praha

4, Czech Republic

2. Laboratory of Systematic Botany and Mycology, University of Tuscia, Largo dell'Università snc, Viterbo 01100, Italy

3. Department of Forest Science and Resources, College of Bioresource Sciences, Nihon University, Fujisawa, Kanagawa, Japan

4. Department of Biology, United Arab Emirates University, Al Ain, Abu Dhabi, United Arab Emirates

corresponding author: Petr Baldrian (baldrian@biomed.cas.cz)

Tomáš Větrovský, Daniel Morais, Petr Kohout and Clémentine Lepinay contributed equally to this work.

\section{Abstract}

Fungi are key players in vital ecosystem services, spanning carbon cycling, decomposition, symbiotic associations with cultural and wild plants and pathogenicity. The high importance of fungi in the ecosystem processes contrasts with the incompleteness of understanding of the patterns of fungal biogeography and the environmental factors that drive it. To close this gap of knowledge, we have here collected and validated data published on the composition of soil fungal communities in terrestrial environments including soil and plant-associated habitats and made them publicly accessible through a user interface at http://globalfungi.com. The GlobalFungi database contains over 650 million observations of fungal sequences across $>20000$ samples with geographical locations and additional metadata contained in 207 original studies with millions of unique sequence variants of the fungal internal transcribed spacers (ITS) 1 and 2 representing fungal species and genera. As it is, the study represents the most comprehensive atlas of fungal distribution on the global scale open to further additions.

\section{Background \& Summary}

Fungi play fundamental roles in the ecosystem processes across all terrestrial biomes. As plant symbionts, pathogens or major decomposers of organic matter they substantially influence plant primary production, carbon mineralization and sequestration, and act as crucial regulators of the soil carbon balance ${ }^{1,2}$. The activities of fungi and their communities contribute to the production of clean water, food, and air and the suppression of disease-causing soil organisms. Soil biodiversity is thus increasingly recognized to provide services critical to food safety and human health ${ }^{3}$. 
It is of high importance to determine how environmental factors affect the diversity and distribution of fungal communities. So far, only a few studies focused on fungal distribution and diversity on global scale ${ }^{4-6}$. Importantly, these single survey studies focused either on a limited number of biomes ${ }^{4,5}$, narrower taxonomical group within the Fungal kingdom ${ }^{6}$, or were restricted only to fungi inhabiting soil. While the benefit of a focused study is the fact that it can rely on standardized sample analysis and metadata collection, its limitation is the inability to cover large sampling efforts in space and time. On the other hand, since the advent of the high-throughput-sequencing methods, large amounts of sequencing data exist for fungi from terrestrial environments with sufficient metadata that allow their evaluation ${ }^{7}$. Recently, the meta-analysis of 36 papers reporting global diversity of soil fungi collected $>3000$ samples and helped to indicate that climate is an important factor for the global distribution of soil fungi and identify the hotspots of fungal diversity outside the tropics ${ }^{8}$. This approach clearly demonstrated the utility of a meta-study approach to address fungal biogeography, ecology and diversity. In addition, the compilation of these data demonstrated the fact that symbiotic mycorrhizal fungi that aid cultural and wild plants to access nutrients, are more likely to be affected by rapid changes of climate than other guilds of fungi, including plant pathogens ${ }^{8}$ and helped to identify which fungi tend to follow alien plants invading new environments ${ }^{9}$.

Here, we have undertaken a comprehensive collection and validation of data published on the composition of soil fungal communities in terrestrial environments including soil and plant-associated habitats. This approach enabled us to construct the GlobalFungi database containing, on March 16, 2020, over 120 million of unique sequence variants ${ }^{10}$ of the fungal internal transcribed spacers (ITS) 1 and 2, covering $>20000$ samples contained in 207 original studies (Figure 1). The dataset of sequence variant frequencies across samples, accompanied by metadata retrieved from published papers and in global climate databases is made publicly available at http://globalfungi.com. To achieve the goal to make published data findable, accessible, interoperable and reusable, the user interface at the above address allows the users to search for individual sequences, fungal species hypotheses ${ }^{11}$, species or genera, to get a visual representation of their distribution in the environment and to access and download sequencing data and metadata. In addition, the user interface also allows authors to submit data from studies not yet covered and in this way to help to build the resource for the community of researchers in mycology, biogeography and ecology of fungi.

\section{Methods}

Data selection. We explored papers that used high-throughput sequencing for the analysis of fungal communities that were published until the beginning of 2019; in total, we explored 843 papers. The following selection criteria were used for the inclusion of samples (and, consequently, studies) into the dataset: (1) samples came from terrestrial biomes of soil, dead or live plant material (e.g., soil, litter, rhizosphere soil, topsoil, lichen, deadwood, root and shoot) and were not subject to experimental treatment; (2) the precise geographic location of each sample was recorded (GPS coordinates); (3) the whole fungal community was subject to amplicon sequencing (studies using group-specific primers were excluded); (4) the internal transcribed spacer regions (ITS1, ITS2, or both) were subject to amplification; (5) sequencing data (either in fasta or fastq format) were publicly available or provided by the authors of the study upon request, and the sequences were unambiguously assigned to samples; (6) the samples could be assigned to biomes according to the Environment Ontology (http://www.ontobee.org/ontology/ENVO) ${ }^{8}$. In total, there were 209 publications contained samples that matched our criteria (Online-Only Table 1).

\section{Processing of sequencing data.}


For the processing of data, see Figure 2. Raw datasets from 207 studies, covering 20009 individual samples were quality filtered by removing all sequences with the mean quality phred scores below 20. Each sequence was labelled using the combination of a sample ID and sequence ID, and the full ITS1 or ITS2 fungal region was extracted using Perl script ITSx v1.0.11 12 . ITS extraction resulted in a total of 416291533 full ITS1 and 231278756 full ITS2 sequences. The extracted ITS sequences were classified according to the representative sequence of the closest UNITE species hypothesis (SH) using BLASTN, considering the SH created using a 98.5\% similarity threshold (general release 8.1 from 2.2.2019 ${ }^{11}$. The sequence was classified to the best best hit SH only when the following thresholds were met: e-value $\left\langle 10 \mathrm{e}^{-50}\right.$, sequence similarity $>=98.5 \%$. All representative sequences annotated as nonfungal were discarded. All representative sequences classified to any fungal $\mathrm{SH}$ and all unclassified sequences were used to build database sequence variant library. The number of sequence variants (unique nucleotide sequences) accessible through the database is 119566 862.

Sample metadata. Sample metadata were collected from the published papers and/or public repositories where they were submitted by the authors. In some cases, metadata were obtained from the authors of individual studies upon request. The samples were assigned to continents, countries or specific locations, and all sites were categorized into biomes following the classification of Environment Ontology to a maximum achievable depth for each sample. The complete list of metadata included in the database is in Table 1.

In addition to metadata provided by the authors of each study, we also extracted bioclimatic variables from the global CHELSA ${ }^{13}$ and WorldClim $2^{14}$ databases for each sample based on its GPS location. Since the results based on CHELSA and WorldClim 2 were comparable, we decided to include those from CHELSA, because precipitation patterns are better captured in the CHELSA dataset, in particular for mountain sites ${ }^{13}$.

For each sequence variant that was classified to $\mathrm{SH}$, fungal species name and genus name was retrieved from the UNITE database ${ }^{11}$, when available.

\section{Data Records}

The raw sequencing reads used to create the database are available at the following locations: One part of the raw ITS sequencing reads can be found from the National Center for Biotechnology Information (NCBI) Sequence Read Archive under accession numbers $\mathrm{SRP} 001058^{15}, \mathrm{SRP}^{2} 01175^{16}, \mathrm{SRP} 006078^{17}, \mathrm{SRP} 012868^{18}{ }^{2}, \mathrm{SRP}^{2} 13695^{19}, \mathrm{SRP}^{2} 13944^{20}$, SRP015735 ${ }^{21}, \mathrm{SRP} 16090^{22}, \mathrm{SRP} 026207^{23}, \mathrm{SRP}^{2} 28404^{24}, \mathrm{SRP}^{2} 33719^{25}, \mathrm{SRP}^{2} 35356^{26}$,

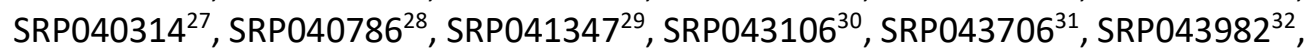
$\mathrm{SRP} 044665^{33}$, SRP045166 ${ }^{34}$, SRP045587 $7^{35}, \mathrm{SRP}^{2} 45746^{36}, \mathrm{SRPO}^{4} 5933^{37}, \mathrm{SRP}^{4} 46049^{38}$,

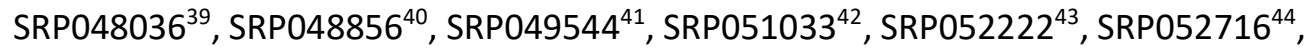
$\mathrm{SRP} 055957^{45}, \mathrm{SRP} 057433^{46}, \mathrm{SRP} 057541^{47}, \mathrm{SRP} 058508^{48}, \mathrm{SRP} 058555^{49}, \mathrm{SRP}^{5} 58851^{50}$,

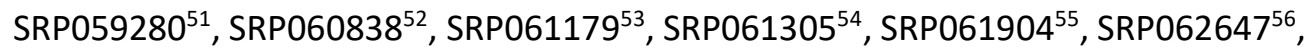

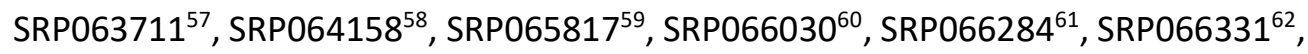

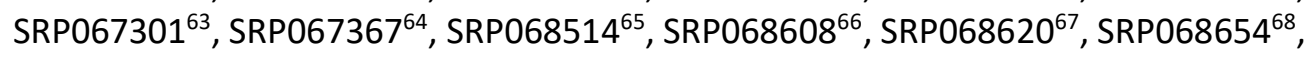

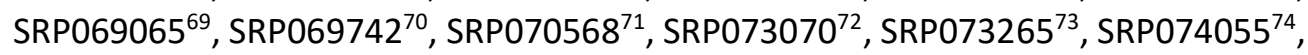
$\mathrm{SRP} 074496^{75}, \mathrm{SRP} 075989^{76}, \mathrm{SRP} 079403^{77}, \mathrm{SRP} 079521^{78}, \mathrm{SRP} 080210^{79}, \mathrm{SRP} 080428^{80}$,

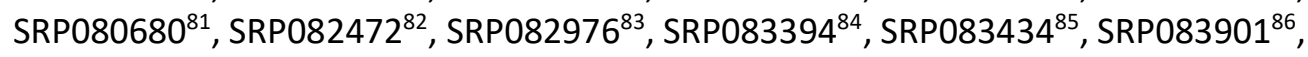
SRP087715 ${ }^{87}$, SRP090261 ${ }^{88}$, SRP090335 $5^{89}$, SRP090490 90 , SRP090651 ${ }^{91}, \mathrm{SRP}^{9} 01741^{92}$,

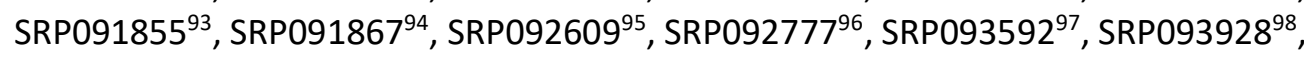
SRP094708 ${ }^{99}$, SRP097883 ${ }^{100}$, SRP101553 $3^{101}$, SRP101605 ${ }^{102}$, SRP102378 103, SRP102417 ${ }^{104}$, SRP102775 $5^{105}$, SRP106137 106, SRP106774 $4^{107}$, SRP107174 108, SRP107743 ${ }^{109}$, SRP109164 ${ }^{110}$,

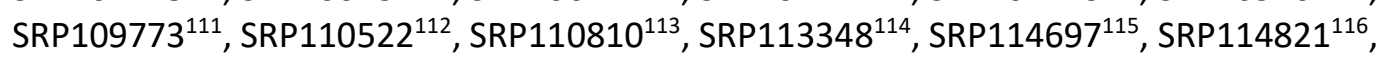

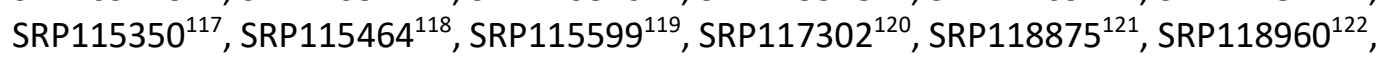


SRP119174 $4^{123}$, SRP125864 124, SRP132277 125, SRP132591 126, SRP132598 127, SRP136886 128

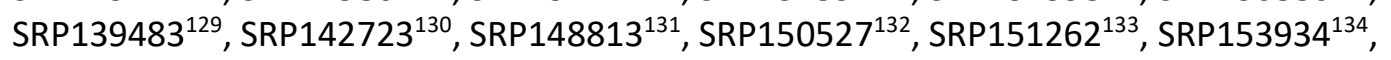
SRP160913 ${ }^{135}$, SRP161632 ${ }^{136}$ and SRP195764 ${ }^{137}$, in the European Nucleotide Archive (ENA) Sequence Read Archive under accession numbers ERP001713 ${ }^{138}$, ERP003251 ${ }^{139}$, ERP003790 ${ }^{140}$, ERP005177 $7^{141}$, ERP005905 ${ }^{142}$, ERP009341 ${ }^{143}, E^{14 P 0} 010027^{144}, E R P 010084^{145}$, ERP010743 ${ }^{146}$, ERP011924 ${ }^{147}$, ERP012017 ${ }^{148}$, ERP013208 ${ }^{149}$, ERP013987 $7^{150}$, ERP014227 $7^{151}$, ERP017480 ${ }^{152}$, ERP017851 ${ }^{153}$, ERP017915 ${ }^{154}$, ERP019580 ${ }^{155}$, ERP019924 ${ }^{156}$, ERP020657 ${ }^{157}$, ERP022511 158, ERP022742 ${ }^{159}$, ERP023275 160, ERP023718 ${ }^{161}$, ERP023855 $5^{162}$, ERP $106131^{163}$, ERP107634 ${ }^{164}$, ERP107636 $6^{165}$, ERP110188 ${ }^{166}$ and ERP112007 $167^{\prime}$, in the DNA Data Bank of Japan (DDBJ) under the accession numbers DRA000926 ${ }^{168}$, DRA000937 $7^{169}$, DRA001737 $7^{170}$, DRA002424 ${ }^{171}$, DRA002469 ${ }^{172}$, DRA003024 ${ }^{173}$, DRA003730 $0^{174}$, DRA004913 ${ }^{175}$, DRA006519 ${ }^{176}$, DRP002783 ${ }^{177}$, DRP003138 ${ }^{178}$ and DRP005365 ${ }^{179}$, in the Dryad Digital Repository under the DOIs https://doi.org/10.5061/dryad.2fc32 $2^{180}$, http://dx.doi.org/10.5061/dryad.n82g9 ${ }^{181}$, http://dx.doi.org/10.5061/dryad.2343k ${ }^{182}$, https://doi.org/10.5061/dryad.gp302 ${ }^{183}$, http://dx.doi.org/10.5061/dryad.cq2rb ${ }^{184}$, https://doi.org/10.5061/dryad.8fn8j ${ }^{185}$ and https://doi.org/10.5061/dryad.216tp ${ }^{186}$, in the MG-RAST repository under the accession numbers $4783710.3^{187}, 4702703.3^{188}, 4524551.3^{189}, 4544233.3^{190}, 4683808.3^{191}$, $4684008.3^{192}, 4696490.3^{193}, 4715213.3^{194}, 4563787.3^{195}, 4563788.3^{196}, 4620497.3^{197}$, $4620498.3^{198}, \mathrm{mgp} 13293^{199}, \mathrm{mgp} 1617^{200}$ and $\mathrm{mgp} 9003^{201}$, in the PlutoF repository under the URLs https://plutof.ut.ee/\#/filerepository/view/1561672 ${ }^{202}$,

https://plutof.ut.ee/\#/filerepository/view/1562683 ${ }^{203}$, https://plutof.ut.ee/\#/doi/10.15156/BIO/100002 ${ }^{204}$ and https://plutof.ut.ee/\#/doi/10.15156/BIO/587446 205 , in the UNITE database under the URL http://unite.ut.ee/454_EcM_CMR.zip ${ }^{206}$, in GenBank under the accessions KAYV00000000.1 $1^{207}$, KAYU00000000.1 $1^{208}$, KAYTO0000000.1 ${ }^{209}$, SAMN02934078 210 and SAMN02934079 211 , in the database of the Australian Antarctic Data Center under DOI http://dx.doi.org/10.4225/15/526f42ada05b $1^{212}$, in the supplemental data of papers under URLs https://staticcontent.springer.com/esm/art\%3A10.1038\%2Fismej.2012.84/MediaObjects/41396_2012_BF ismej201284_MOESM78_ESM.zip ${ }^{213}$ and https://staticcontent.springer.com/esm/art\%3A10.1038\%2Fismej.2015.238/MediaObjects/41396_2016_ BFismej2015238_MOESM67_ESM.zip ${ }^{214}$, and on server of the Czech Academy of Sciences under URLs www.biomed.cas.cz/mbu/lbwrf/metastudy_datasets/GF0001.zip ${ }^{215}$, www.biomed.cas.cz/mbu/lbwrf/metastudy_datasets/GF0002.zip ${ }^{216}$, www.biomed.cas.cz/mbu/lbwrf/metastudy_datasets/GF0003.zip ${ }^{217}$, www.biomed.cas.cz/mbu/lbwrf/metastudy_datasets/GF0004.zip ${ }^{218}$, www.biomed.cas.cz/mbu/lbwrf/metastudy_datasets/GF0005.zip ${ }^{219}$, www.biomed.cas.cz/mbu/lbwrf/metastudy_datasets/GF0006.zip ${ }^{220}$, www.biomed.cas.cz/mbu/lbwrf/metastudy_datasets/GF0007.zip ${ }^{221}$, www.biomed.cas.cz/mbu/lbwrf/metastudy_datasets/GF0008.zip ${ }^{222}$, www.biomed.cas.cz/mbu/lbwrf/metastudy_datasets/GF0009.zip ${ }^{223}$, www.biomed.cas.cz/mbu/lbwrf/metastudy_datasets/GF0010.zip ${ }^{224}$, www.biomed.cas.cz/mbu/lbwrf/metastudy_datasets/GF0011.zip ${ }^{225}$, www.biomed.cas.cz/mbu/lbwrf/metastudy_datasets/GF0012.zip ${ }^{226}$.

The database contains two data types: sequence variants (individual nucleotide sequences) and samples. For each sequence variant, the following information is stored: sequence variant code, identification of samples where sequence variant occurs and the number of observations, the SH of best hit (when available), fungal species name (when available), fungal genus name (when available). For each sample, metadata information is stored (Table 1). Sequencing data and metadata are accessible at Figshare (GlobalFungi_ITS_variants.zip, GlobalFungi_metadata.xIsx). All database content is accessible using a public graphical user interface at http://globalfungi.com. 


\section{Technical Validation}

The technical validation included the screening of the data sources, sequencing data and data reliability. Regarding the data source screening, the data sources (published papers) were screened to fulfil the criteria outlined in the Methods section. The dataset was thoroughly checked for duplicities and for all records that appeared in multiple publications, only the first original publication of the dataset was considered as a datasource. Considering sequence quality, we have only utilized those primer pairs that are generally accepted to target general fungi ${ }^{7,227}$. Sequences were quality filtered by removing all sequences with the mean quality phred scores below 20 and sequences that did not represent complete ITS1 or ITS2 after extraction or those that were identified as chimeric by the ITS extraction software ${ }^{12}$ were removed. All representative sequences where the BLASTn search against the UNITE database ${ }^{11}$ resulted in a nonfungal organism, were discarded.

For data reliability, the geographic location represented by the GPS coordinates was validated first. For each sample set, the geographic location of the sample described in the text of the study was confronted with the location on the map. For those samples, where disagreement was recorded (e.g., terrestrial samples positioned in the ocean, location in another region than described in the text), the authors of each study were asked for correction. If impossible, such samples were deleted. The quality of sample metadata was checked and if they were outside the acceptable range (such as content of elements or organic matter $>100 \%$ ), these invalid metadata were removed.

\section{Usage Notes}

The user interface at http://globalfungi.com enables the users to access the database in several ways (Figure 3). In taxon search, it is possible to seach for genera and species of fungi and for the $98.5 \%$ SH species hypotheses of UNITE, contained in the general release 8.1 from 2.2.2019. The search results open the options to download corresponding $\mathrm{SH}$ or corresponding sequence variants. It is also possible to view breakdown of samples by type, biome, mean annual temperature, mean annual precipitation, $\mathrm{pH}$ and continents. The results also contain an interactive map of the taxon distribution with relative abundances of sequences of the taxon across samples and a list of samples with metadata. Several modes of filtering of results are available as well.

In sequence search, it is possible to search for multiple nucleotide sequences by exact match or BLAST retrieving the sequence variant best hit in the database, and alternatively, blasting a single sequence can deliver multiple ranked high score hits among the sequence variants. It is also possible to open individual studies and access their content and to select a group of samples on the map with a range of tools and retrieve data for these samples (such as the FASTA file with all occurring sequence variants).

Importantly, the database is intended to grow, both by the continuing activity of the authors and using the help of the scientific community. For that, web interface enabling the submission of studies not yet represented is available to users. The submission tool guides the submitting person through the steps where details about the publication, samples, sample metadata and sequences are sequentially collected. The submitted data will be added to the existing dataset after processing and validation by the authors.

\section{Code Availability}

The workflow included several custom made python scripts (labelled by star) which are accessible here: https://github.com/VetrovskyTomas/GlobalFungi. 


\section{Acknowledgements}

We acknowledge funding from the Czech Science Foundation Grant No 18-26191S. ELIXIR CZ research infrastructure project LM2015047 by the Ministry of Education, Youth and Sports of the Czech Republic is acknowledged for hosting the database. All corresponding authors of published studies that provided additional information on the samples included in the database are gratefully acknowledged.

\section{Author contributions}

T.V., P.K. and P.B. jointly conceived the study. C.L. and coordinated data acquisition, T.V., D.M., M.K. and P.B. designed the database, T.V. and D.M. developed the database and created the user interface. C.A.G., S.A.H., B.D.B., K.B., V.B., F.D., R.Z.H., M.J., J.K., C.L., S.L., R.L.M., T.Mar., T.Maš., L.Me., L.Mi., T.Mi., S.M., D.N., I.O., S.P.C., M.Š., K.Š., V.T., M.U., L.V., J.V. and L.Ž. identified data sources, processed and analysed sequencing information, collated and analysed metadata. P.B. drafted the manuscript along with T.V., C.L. and P.K. All authors wrote and reviewed the manuscript.

T.V., D.M., P.K. and C.L. contributed equally to this work.

\section{Competing interests}

The authors declare no competing interests.

\section{References}

1. Crowther, T.W., Todd-Brown, K.E.O., Rowe, C.W., Wieder, W.R., Carey, J.C., Machmuller, M.B., Snoek, B.L., Fang, S., Zhou, G., Allison, S.D., Blair, J.M., Bridgham, S.D., Burton, A.J., Carrillo, Y., Reich, P.B., Clark, J.S., Classen, A.T., Dijkstra, F.A., Elberling, B., Emmett, B.A., Estiarte, M., Frey, S.D., Guo, J., Harte, J., Jiang, L., Johnson, B.R., Kroel-Dulay, G., Larsen, K.S., Laudon, H., Lavallee, J.M., Luo, Y., Lupascu, M., Ma, L.N., Marhan, S., Michelsen, A., Mohan, J., Niu, S., Pendall, E., Penuelas, J., Pfeifer-Meister, L., Poll, C., Reinsch, S., Reynolds, L.L., Schmidt, I.K., Sistla, S., Sokol, N.W., Templer, P.H., Treseder, K.K., Welker, J.M. \& Bradford, M.A. Quantifying global soil carbon losses in response to warming. Nature 540, 104-108 (2016).

2. Peay, K.G., Kennedy, P.G. \& Talbot, J.M. Dimensions of biodiversity in the Earth mycobiome. Nature Rev. Microbiol. 14, 434-447 (2016).

3. Wall, D.H., Nielsen, U.N. \& Six, J. Soil biodiversity and human health. Nature 528, 69-76 (2015).

4. Tedersoo, L., Bahram, M., Polme, S., Koljalg, U., Yorou, N.S., Wijesundera, R., Ruiz, L.V., Vasco-Palacios, A.M., Thu, P.Q., Suija, A., Smith, M.E., Sharp, C., Saluveer, E., Saitta, A., Rosas, M., Riit, T., Ratkowsky, D., Pritsch, K., Poldmaa, K., Piepenbring, M., Phosri, C., Peterson, M., Parts, K., Partel, K., Otsing, E., Nouhra, E., Njouonkou, A.L., Nilsson, R.H., Morgado, L.N., Mayor, J., May, T.W., Majuakim, L., Lodge, D.J., Lee, S.S., Larsson, K.H., Kohout, P., Hosaka, K., Hiiesalu, I., Henkel, T.W., Harend, H., Guo, L.D., Greslebin, A., Grelet, G., Geml, J., Gates, G., Dunstan, W., Dunk, C., Drenkhan, R., Dearnaley, J., De Kesel, A., Dang, T., Chen, X., Buegger, F., Brearley, F.Q., Bonito, G., Anslan, S., Abell, S. \& Abarenkov, K. Global diversity and geography of soil fungi. Science 346, 1256688 (2014).

5. Bahram, M., Hildebrand, F., Forslund, S.K., Anderson, J.L., Soudzilovskaia, N.A., Bodegom, P.M., Bengtsson-Palme, J., Anslan, S., Coelho, L.P., Harend, H., Huerta-Cepas, J., Medema, M.H., Maltz, M.R., Mundra, S., Olsson, P.A., Pent, M., Polme, S., Sunagawa, S., Ryberg, M., 
Tedersoo, L. \& Bork, P. Structure and function of the global topsoil microbiome. Nature 560, 233-237 (2018).

6. Egidi, E., Delgado-Baquerizo, M., Plett, J.M., Wang, J., Eldridge, D.J., Bardgett, R.D., Maestre, F.T. \& Singh, B.K. A few Ascomycota taxa dominate soil fungal communities worldwide. Nature Commun. 10, 2369 (2019).

7. Nilsson, R.H., Anslan, S., Bahram, M., Wurzbacher, C., Baldrian, P. \& Tedersoo, L. Mycobiome diversity: high-throughput sequencing and identification of fungi. Nature Rev. Microbiol. 17, 95-109 (2019).

8. Větrovský, T., Kohout, P., Kopecký, M., Machac, A., Man, M., Bahnmann, B.D., Brabcová, V., Choi, J., Meszárošová, L., Human, Z.R., Lepinay, C., Lladó, S., Lopez-Mondejar, R., Martinovic, T., Mašínová, T., Morais, D., Navrátilová, D., Odriozola, I., Štursová, M., Švec, K., Tláskal, V., Urbanová, M., Wan, J., Žifčáková, L., Howe, A., Ladau, J., Peay, K.G., Storch, D., Wild, J. \& Baldrian, P. A meta-analysis of global fungal distribution reveals climate-driven patterns. Nature Commun. 10, 5142 (2019).

9. VIk, L., Tedersoo, L., Antl, T., Větrovský, T., Abarenkov, K., Pergl, J., Albrechtová, J., Vosátka, M., Baldrian, P., Pyšek, P. \& Kohout, P. Early successional ectomycorrhizal fungi are more likely to naturalize outside their native range than other ectomycorrhizal fungi. New Phytol. in press, doi: 10.1111/nph.16557.

10. Thompson, L.R., Sanders, J.G., McDonald, D., Amir, A., Ladau, J., Locey, K.J., Prill, R.J., Tripathi, A., Gibbons, S.M., Ackermann, G., Navas-Molina, J.A., Janssen, S., Kopylova, E., Vázquez-Baeza, Y., González, A., Morton, J.T., Mirarab, S., Zech Xu, Z., Jiang, L., Haroon, M.F., Kanbar, J., Zhu, Q., Jin Song, S., Kosciolek, T., Bokulich, N.A., Lefler, J., Brislawn, C.J., Humphrey, G., Owens, S.M., Hampton-Marcell, J., Berg-Lyons, D., McKenzie, V., Fierer, N., Fuhrman, J.A., Clauset, A., Stevens, R.L., Shade, A., Pollard, K.S., Goodwin, K.D., Jansson, J.K., Gilbert, J.A. Knight, R. \& The Earth Microbiome Project, C. A communal catalogue reveals Earth's multiscale microbial diversity. Nature 551, 457-463 (2017).

11. Nilsson, R.H., Larsson, K.H., Taylor, A.F.S., Bengtsson-Palme, J., Jeppesen, T.S., Schigel, D., Kennedy, P., Picard, K., Glockner, F.O., Tedersoo, L., Saar, I., Koljalg, U. \& Abarenkov, K. The UNITE database for molecular identification of fungi: handling dark taxa and parallel taxonomic classifications. Nucleic Acids Res. 47, D259-D264 (2019).

12. Bengtsson-Palme, J., Ryberg, M., Hartmann, M., Branco, S., Wang, Z., Godhe, A., De Wit, P., Sanchez-Garcia, M., Ebersberger, I., de Sousa, F., Amend, A.S., Jumpponen, A., Unterseher, M., Kristiansson, E., Abarenkov, K., Bertrand, Y.J.K., Sanli, K., Eriksson, K.M., Vik, U., Veldre, V. \& Nilsson, R.H. Improved software detection and extraction of ITS1 and ITS2 from ribosomal ITS sequences of fungi and other eukaryotes for analysis of environmental sequencing data. Meth. Ecol. Evol. 4, 914-919 (2013).

13. Karger, D.N., Conrad, O., Bohner, J., Kawohl, T., Kreft, H., Soria-Auza, R.W., Zimmermann, N.E., Linder, H.P. \& Kessler, M. Data Descriptor: Climatologies at high resolution for the earth's land surface areas. Scientific Data 4, 170122 (2017).

14. Fick, S.E. \& Hijmans, R.J. WorldClim 2: new 1-km spatial resolution climate surfaces for global land areas. International J. Climatol. 37, 4302-4315 (2017).

15. NCBI Sequence Read Archive, https://identifiers.org/ncbi/insdc.sra:SRP001058 (2010). 16. NCBI Sequence Read Archive, https://identifiers.org/ncbi/insdc.sra:SRP001175 (2010). 17. NCBI Sequence Read Archive, https://identifiers.org/ncbi/insdc.sra:SRP006078 (2011). 18. NCBI Sequence Read Archive, https://identifiers.org/ncbi/insdc.sra:SRP012868 (2012). 19. NCBI Sequence Read Archive, https://identifiers.org/ncbi/insdc.sra:SRP013695 (2012). 20. NCBI Sequence Read Archive, https://identifiers.org/ncbi/insdc.sra:SRP013944 (2016). 21. NCBI Sequence Read Archive, https://identifiers.org/ncbi/insdc.sra:SRP015735 (2015). 22. NCBI Sequence Read Archive, https://identifiers.org/ncbi/insdc.sra:SRP016090 (2015).

23. NCBI Sequence Read Archive, https://identifiers.org/ncbi/insdc.sra:SRP026207 (2014).

24. NCBI Sequence Read Archive, https://identifiers.org/ncbi/insdc.sra:SRP028404 (2015).

25. NCBI Sequence Read Archive, https://identifiers.org/ncbi/insdc.sra:SRP033719 (2015). 
26. NCBI Sequence Read Archive, https://identifiers.org/ncbi/insdc.sra:SRP035356 (2015). 27. NCBI Sequence Read Archive, https://identifiers.org/ncbi/insdc.sra:SRP040314 (2014). 28. NCBI Sequence Read Archive, https://identifiers.org/ncbi/insdc.sra:SRP040786 (2015). 29. NCBI Sequence Read Archive, https://identifiers.org/ncbi/insdc.sra:SRP041347 (2015). 30. NCBI Sequence Read Archive, https://identifiers.org/ncbi/insdc.sra:SRP043106 (2015). 31. NCBI Sequence Read Archive, https://identifiers.org/ncbi/insdc.sra:SRP043706 (2017). 32. NCBI Sequence Read Archive, https://identifiers.org/ncbi/insdc.sra:SRP043982 (2015). 33. NCBI Sequence Read Archive, https://identifiers.org/ncbi/insdc.sra:SRP044665 (2016). 34. NCBI Sequence Read Archive, https://identifiers.org/ncbi/insdc.sra:SRP045166 (2015). 35. NCBI Sequence Read Archive, https://identifiers.org/ncbi/insdc.sra:SRP045587 (2016). 36. NCBI Sequence Read Archive, https://identifiers.org/ncbi/insdc.sra:SRP045746 (2014). 37. NCBI Sequence Read Archive, https://identifiers.org/ncbi/insdc.sra:SRP045933 (2015). 38. NCBI Sequence Read Archive, https://identifiers.org/ncbi/insdc.sra:SRP046049 (2016). 39. NCBI Sequence Read Archive, https://identifiers.org/ncbi/insdc.sra:SRP048036 (2016). 40. NCBI Sequence Read Archive, https://identifiers.org/ncbi/insdc.sra:SRP048856 (2015). 41. NCBI Sequence Read Archive, https://identifiers.org/ncbi/insdc.sra:SRP049544 (2015). 42. NCBI Sequence Read Archive, https://identifiers.org/ncbi/insdc.sra:SRP051033 (2016). 43. NCBI Sequence Read Archive, https://identifiers.org/ncbi/insdc.sra:SRP052222 (2017). 44. NCBI Sequence Read Archive, https://identifiers.org/ncbi/insdc.sra:SRP052716 (2015). 45. NCBI Sequence Read Archive, https://identifiers.org/ncbi/insdc.sra:SRP055957 (2015). 46. NCBI Sequence Read Archive, https://identifiers.org/ncbi/insdc.sra:SRP057433 (2016). 47. NCBI Sequence Read Archive, https://identifiers.org/ncbi/insdc.sra:SRP057541 (2016). 48. NCBI Sequence Read Archive, https://identifiers.org/ncbi/insdc.sra:SRP058508 (2016). 49. NCBI Sequence Read Archive, https://identifiers.org/ncbi/insdc.sra:SRP058555 (2016). 50. NCBI Sequence Read Archive, https://identifiers.org/ncbi/insdc.sra:SRP058851 (2018). 51. NCBI Sequence Read Archive, https://identifiers.org/ncbi/insdc.sra:SRP059280 (2016). 52. NCBI Sequence Read Archive, https://identifiers.org/ncbi/insdc.sra:SRP060838 (2016). 53. NCBI Sequence Read Archive, https://identifiers.org/ncbi/insdc.sra:SRP061179 (2016). 54. NCBI Sequence Read Archive, https://identifiers.org/ncbi/insdc.sra:SRP061305 (2017). 55. NCBI Sequence Read Archive, https://identifiers.org/ncbi/insdc.sra:SRP061904 (2015). 56. NCBI Sequence Read Archive, https://identifiers.org/ncbi/insdc.sra:SRP062647 (2016). 57. NCBI Sequence Read Archive, https://identifiers.org/ncbi/insdc.sra:SRP063711 (2017). 58. NCBI Sequence Read Archive, https://identifiers.org/ncbi/insdc.sra:SRP064158 (2017). 59. NCBI Sequence Read Archive, https://identifiers.org/ncbi/insdc.sra:SRP065817 (2017). 60. NCBI Sequence Read Archive, https://identifiers.org/ncbi/insdc.sra:SRP066030 (2016). 61. NCBI Sequence Read Archive, https://identifiers.org/ncbi/insdc.sra:SRP066284 (2017). 62. NCBI Sequence Read Archive, https://identifiers.org/ncbi/insdc.sra:SRP066331 (2017). 63. NCBI Sequence Read Archive, https://identifiers.org/ncbi/insdc.sra:SRP067301 (2017). 64. NCBI Sequence Read Archive, https://identifiers.org/ncbi/insdc.sra:SRP067367 (2016). 65. NCBI Sequence Read Archive, https://identifiers.org/ncbi/insdc.sra:SRP068514 (2016). 66. NCBI Sequence Read Archive, https://identifiers.org/ncbi/insdc.sra:SRP068608 (2016). 67. NCBI Sequence Read Archive, https://identifiers.org/ncbi/insdc.sra:SRP068620 (2016). 68. NCBI Sequence Read Archive, https://identifiers.org/ncbi/insdc.sra:SRP068654 (2016). 69. NCBI Sequence Read Archive, https://identifiers.org/ncbi/insdc.sra:SRP069065 (2017). 70. NCBI Sequence Read Archive, https://identifiers.org/ncbi/insdc.sra:SRP069742 (2017). 71. NCBI Sequence Read Archive, https://identifiers.org/ncbi/insdc.sra:SRP070568 (2016). 72. NCBI Sequence Read Archive, https://identifiers.org/ncbi/insdc.sra:SRP073070 (2016). 73. NCBI Sequence Read Archive, https://identifiers.org/ncbi/insdc.sra:SRP073265 (2017). 74. NCBI Sequence Read Archive, https://identifiers.org/ncbi/insdc.sra:SRP074055 (2016). 75. NCBI Sequence Read Archive, https://identifiers.org/ncbi/insdc.sra:SRP074496 (2016). 76. NCBI Sequence Read Archive, https://identifiers.org/ncbi/insdc.sra:SRP075989 (2017). 77. NCBI Sequence Read Archive, https://identifiers.org/ncbi/insdc.sra:SRP079403 (2017). 
78. NCBI Sequence Read Archive, https://identifiers.org/ncbi/insdc.sra:SRP079521 (2018). 79. NCBI Sequence Read Archive, https://identifiers.org/ncbi/insdc.sra:SRP080210 (2016). 80. NCBI Sequence Read Archive, https://identifiers.org/ncbi/insdc.sra:SRP080428 (2017). 81. NCBI Sequence Read Archive, https://identifiers.org/ncbi/insdc.sra:SRP080680 (2017). 82. NCBI Sequence Read Archive, https://identifiers.org/ncbi/insdc.sra:SRP082472 (2017). 83. NCBI Sequence Read Archive, https://identifiers.org/ncbi/insdc.sra:SRP082976 (2017). 84. NCBI Sequence Read Archive, https://identifiers.org/ncbi/insdc.sra:SRP083394 (2017). 85. NCBI Sequence Read Archive, https://identifiers.org/ncbi/insdc.sra:SRP083434 (2017). 86. NCBI Sequence Read Archive, https://identifiers.org/ncbi/insdc.sra:SRP083901 (2017). 87. NCBI Sequence Read Archive, https://identifiers.org/ncbi/insdc.sra:SRP087715 (2017). 88. NCBI Sequence Read Archive, https://identifiers.org/ncbi/insdc.sra:SRP090261 (2016). 89. NCBI Sequence Read Archive, https://identifiers.org/ncbi/insdc.sra:SRP090335 (2017). 90. NCBI Sequence Read Archive, https://identifiers.org/ncbi/insdc.sra:SRP090490 (2017). 91. NCBI Sequence Read Archive, https://identifiers.org/ncbi/insdc.sra:SRP090651 (2017). 92. NCBI Sequence Read Archive, https://identifiers.org/ncbi/insdc.sra:SRP091741 (2017). 93. NCBI Sequence Read Archive, https://identifiers.org/ncbi/insdc.sra:SRP091855 (2018). 94. NCBI Sequence Read Archive, https://identifiers.org/ncbi/insdc.sra:SRP091867 (2016). 95. NCBI Sequence Read Archive, https://identifiers.org/ncbi/insdc.sra:SRP092609 (2019). 96. NCBI Sequence Read Archive, https://identifiers.org/ncbi/insdc.sra:SRP092777 (2017). 97. NCBI Sequence Read Archive, https://identifiers.org/ncbi/insdc.sra:SRP093592 (2017). 98. NCBI Sequence Read Archive, https://identifiers.org/ncbi/insdc.sra:SRP093928 (2017). 99. NCBI Sequence Read Archive, https://identifiers.org/ncbi/insdc.sra:SRP094708 (2017). 100. NCBI Sequence Read Archive, https://identifiers.org/ncbi/insdc.sra:SRP097883 (2017). 101. NCBI Sequence Read Archive, https://identifiers.org/hcbi/insdc.sra:SRP101553 (2017). 102. NCBI Sequence Read Archive, https://identifiers.org/hcbi/insdc.sra:SRP101605 (2018). 103. NCBI Sequence Read Archive, https://identifiers.org/ncbi/insdc.sra:SRP102378 (2017). 104. NCBI Sequence Read Archive, https://identifiers.org/ncbi/insdc.sra:SRP102417 (2018). 105. NCBI Sequence Read Archive, https://identifiers.org/ncbi/insdc.sra:SRP102775 (2018). 106. NCBI Sequence Read Archive, https://identifiers.org/hcbi/insdc.sra:SRP106137 (2018). 107. NCBI Sequence Read Archive, https://identifiers.org/ncbi/insdc.sra:SRP106774 (2018). 108. NCBI Sequence Read Archive, https://identifiers.org/ncbi/insdc.sra:SRP107174 (2017). 109. NCBI Sequence Read Archive, https://identifiers.org/hcbi/insdc.sra:SRP107743 (2017). 110. NCBI Sequence Read Archive, https://identifiers.org/ncbi/insdc.sra:SRP109164 (2017). 111. NCBI Sequence Read Archive, https://identifiers.org/ncbi/insdc.sra:SRP109773 2017). 112. NCBI Sequence Read Archive, https://identifiers.org/ncbi/insdc.sra:SRP110522 (2017). 113. NCBI Sequence Read Archive, https://identifiers.org/hcbi/insdc.sra:SRP110810 (2017). 114. NCBI Sequence Read Archive, https://identifiers.org/ncbi/insdc.sra:SRP113348 (2018). 115. NCBI Sequence Read Archive, https://identifiers.org/hcbi/insdc.sra:SRP114697 (2017). 116. NCBI Sequence Read Archive, https://identifiers.org/ncbi/insdc.sra:SRP114821 (2018). 117. NCBI Sequence Read Archive, https://identifiers.org/hcbi/insdc.sra:SRP115350 (2018). 118. NCBI Sequence Read Archive, https://identifiers.org/ncbi/insdc.sra:SRP115464 (2018). 119. NCBI Sequence Read Archive, https://identifiers.org/ncbi/insdc.sra:SRP115599 (2018). 120. NCBI Sequence Read Archive, https://identifiers.org/hcbi/insdc.sra:SRP117302 (2018). 121. NCBI Sequence Read Archive, https://identifiers.org/hcbi/insdc.sra:SRP118875 (2018). 122. NCBI Sequence Read Archive, https://identifiers.org/ncbi/insdc.sra:SRP118960 (2018). 123. NCBI Sequence Read Archive, https://identifiers.org/hcbi/insdc.sra:SRP119174 (2017). 124. NCBI Sequence Read Archive, https://identifiers.org/ncbi/insdc.sra:SRP125864 (2016). 125. NCBI Sequence Read Archive, https://identifiers.org/hcbi/insdc.sra:SRP132277 (2018). 126. NCBI Sequence Read Archive, https://identifiers.org/hcbi/insdc.sra:SRP132591 (2018). 127. NCBI Sequence Read Archive, https://identifiers.org/hcbi/insdc.sra:SRP132598 (2018). 128. NCBI Sequence Read Archive, https://identifiers.org/ncbi/insdc.sra:SRP136886 (2012). 129. NCBI Sequence Read Archive, https://identifiers.org/ncbi/insdc.sra:SRP139483 (2019). 
130. NCBI Sequence Read Archive, https://identifiers.org/ncbi/insdc.sra:SRP142723 (2018).

131. NCBI Sequence Read Archive, https://identifiers.org/ncbi/insdc.sra:SRP148813 (2018).

132. NCBI Sequence Read Archive, https://identifiers.org/ncbi/insdc.sra:SRP150527 (2019).

133. NCBI Sequence Read Archive, https://identifiers.org/ncbi/insdc.sra:SRP151262 (2018).

134. NCBI Sequence Read Archive, https://identifiers.org/ncbi/insdc.sra:SRP153934 (2018).

135. NCBI Sequence Read Archive, https://identifiers.org/hcbi/insdc.sra:SRP160913 (2018).

136. NCBI Sequence Read Archive, https://identifiers.org/ncbi/insdc.sra:SRP161632 (2018).

137. NCBI Sequence Read Archive, https://identifiers.org/ncbi/insdc.sra:SRP195764 (2019).

138. European Nucleotide Archive, https://identifiers.org/ena.embl:ERP001713 (2014).

139. European Nucleotide Archive, https://identifiers.org/ena.embl:ERP003251 (2013).

140. European Nucleotide Archive, https://identifiers.org/ena.embl:ERP003790 (2015).

141. European Nucleotide Archive, https://identifiers.org/ena.embl:ERP005177 (2015).

142. European Nucleotide Archive, https://identifiers.org/ena.embl:ERP005905 (2015).

143. European Nucleotide Archive, https://identifiers.org/ena.embl:ERP009341 (2015).

144. European Nucleotide Archive, https://identifiers.org/ena.embl:ERP010027 (2017).

145. European Nucleotide Archive, https://identifiers.org/ena.embl:ERP010084 (2016).

146. European Nucleotide Archive, https://identifiers.org/ena.embl:ERP010743 (2016).

147. European Nucleotide Archive, https://identifiers.org/ena.embl:ERP011924 (2016).

148. European Nucleotide Archive, https://identifiers.org/ena.embl:ERP012017 (2016).

149. European Nucleotide Archive, https://identifiers.org/ena.embl:ERP013208 (2016).

150. European Nucleotide Archive, https://identifiers.org/ena.embl:ERP013987 (2017).

151. European Nucleotide Archive, https://identifiers.org/ena.embl:ERP014227 (2016).

152. European Nucleotide Archive, https://identifiers.org/ena.embl:ERP017480 (2018).

153. European Nucleotide Archive, https://identifiers.org/ena.embl:ERP017851 (2017).

154. European Nucleotide Archive, https://identifiers.org/ena.embl:ERP017915 (2017).

155. European Nucleotide Archive, https://identifiers.org/ena.embl:ERP019580 (2017).

156. European Nucleotide Archive, https://identifiers.org/ena.embl:ERP019924 (2017).

157. European Nucleotide Archive, https://identifiers.org/ena.embl:ERP020657 (2017).

158. European Nucleotide Archive, https://identifiers.org/ena.embl:ERP022511 (2019).

159. European Nucleotide Archive, https://identifiers.org/ena.embl:ERP022742 (2017).

160. European Nucleotide Archive, https://identifiers.org/ena.embl:ERP023275 (2018).

161. European Nucleotide Archive, https://identifiers.org/ena.embl:ERP023718 (2018).

162. European Nucleotide Archive, https://identifiers.org/ena.embl:ERP023855 (2018).

163. European Nucleotide Archive, https://identifiers.org/ena.embl:ERP106131 (2018).

164. European Nucleotide Archive, https://identifiers.org/ena.embl:ERP107634 (2019).

165. European Nucleotide Archive, https://identifiers.org/ena.embl:ERP107636 (2019).

166. European Nucleotide Archive, https://identifiers.org/ena.embl:ERP110188 (2019).

167. European Nucleotide Archive, https://identifiers.org/ena.embl:ERP112007 (2019).

168. DNA Data Bank of Japan,

https://trace.ddbj.nig.ac.jp/DRASearch/submission?acc=DRA000926 (2014).

169. DNA Data Bank of Japan,

https://trace.ddbj.nig.ac.jp/DRASearch/submission?acc=DRA000937 (2014).

170. DNA Data Bank of Japan,

https://trace.ddbj.nig.ac.jp/DRASearch/submission?acc=DRA001737 (2016).

171. DNA Data Bank of Japan,

https://trace.ddbj.nig.ac.jp/DRASearch/submission?acc=DRA002424 (2016).

172. DNA Data Bank of Japan,

https://trace.ddbj.nig.ac.jp/DRASearch/submission?acc=DRA002469 (2016).

173. DNA Data Bank of Japan,

https://trace.ddbj.nig.ac.jp/DRASearch/submission?acc=DRA003024 (2016).

174. DNA Data Bank of Japan,

https://trace.ddbj.nig.ac.jp/DRASearch/submission?acc=DRA003730 (2016). 
175. DNA Data Bank of Japan, https://trace.ddbj.nig.ac.jp/DRASearch/submission?acc=DRA004913 (2017). 176. DNA Data Bank of Japan, https://trace.ddbj.nig.ac.jp/DRASearch/submission?acc=DRA006519 (2018). 177. DNA Data Bank of Japan, https://trace.ddbj.nig.ac.jp/DRASearch/study?acc=DRP002783 (2015).

178. DNA Data Bank of Japan, https://trace.ddbj.nig.ac.jp/DRASearch/study?acc=DRP003138 (2016).

179. DNA Data Bank of Japan, https://trace.ddbj.nig.ac.jp/DRASearch/study?acc=DRP005365 (2019).

180. Dryad Digital Repository, https://doi.org/10.5061/dryad.2fc32 (2015).

181. Dryad Digital Repository, http://dx.doi.org/10.5061/dryad.n82g9 (2017).

182. Dryad Digital Repository, http://dx.doi.org/10.5061/dryad.2343k (2015).

183. Dryad Digital Repository, https://doi.org/10.5061/dryad.gp302 (2015).

184. Dryad Digital Repository, http://dx.doi.org/10.5061/dryad.cq2rb (2016).

185. Dryad Digital Repository, https://doi.org/10.5061/dryad.8fn8j (2017).

186. Dryad Digital Repository, https://doi.org/10.5061/dryad.216tp (2013).

187. MG-RAST, https://www.mg-rast.org/mgmain.html?mgpage=search\&search $=4783710.3$ (2012).

188. MG-RAST, https://www.mg-rast.org/mgmain.html?mgpage=search\&search=4702703.3 (2016).

189. MG-RAST, https://www.mg-rast.org/mgmain.html?mgpage=search\&search=4524551.3 (2014).

190. MG-RAST, https://www.mg-rast.org/mgmain.html?mgpage=search\&search=4544233.3 (2016).

191. MG-RAST, https://www.mg-rast.org/mgmain.html?mgpage=search\&search=4683808.3 (2017).

192. MG-RAST, https://www.mg-rast.org/mgmain.html?mgpage=search\&search=4684008.3 (2016).

193. MG-RAST, https://www.mg-rast.org/mgmain.html?mgpage=search\&search=4696490.3 (2016).

194. MG-RAST, https://www.mg-rast.org/mgmain.html?mgpage=search\&search=4715213.3 (2016).

195. MG-RAST, https://www.mg-rast.org/mgmain.html?mgpage=search\&search=4563787.3 (2015).

196. MG-RAST, https://www.mg-rast.org/mgmain.html?mgpage=search\&search=4563788.3 (2015).

197. MG-RAST, https://www.mg-rast.org/mgmain.html?mgpage=search\&search=4620497.3 (2015).

198. MG-RAST, https://www.mg-rast.org/mgmain.html?mgpage=search\&search=4620498.3 (2015).

199. MG-RAST, https://www.mg-rast.org/mgmain.html?mgpage=search\&search=mgp13293 (2012).

200. MG-RAST, https://www.mg-rast.org/mgmain.html?mgpage=search\&search=mgp1617 (2013).

201. MG-RAST, https://www.mg-rast.org/mgmain.html?mgpage=search\&search=mgp9003 (2014).

202. PlutoF repository, https://plutof.ut.ee/\#/filerepository/view/1561672 (2017).

203. PlutoF repository, https://plutof.ut.ee/\#/filerepository/view/1562683 (2017).

204. PlutoF repository, https://plutof.ut.ee/\#/doi/10.15156/BIO/100002 (2015).

205. PlutoF repository, https://plutof.ut.ee/\#/doi/10.15156/BIO/587446 (2017).

206. UNITE database, http://unite.ut.ee/454 EcM CMR.zip (2010). 
207. Schappe, T., Albornoz, F.E., Turner, B.L., Neat, A., Condit, R. and Jones, F.A. TLS: uncultured fungus internal transcribed spacer 1 , targeted locus study. Genbank https://identifiers.org/ncbi/insdc:KAYV00000000.1 (2017).

208. Schappe, T., Albornoz, F.E., Turner, B.L., Neat, A., Condit, R. and Jones, F.A. TLS: uncultured fungus internal transcribed spacer 1, targeted locus study. Genbank https://identifiers.org/ncbi/insdc:KAYU00000000.1 (2017).

209. Schappe, T., Albornoz, F.E., Turner, B.L., Neat, A., Condit, R. and Jones, F.A. TLS: uncultured fungus internal transcribed spacer 1 , targeted locus study. Genbank https://identifiers.org/ncbi/insdc:KAYT00000000.1 (2017).

210. Vaz, A.B., Fonseca, P.L., Leite, L.R., Badotti, F., Salim, A.C., Araujo, F.M., CuadrosOrellana, S., Duarte, A.A., Rosa, C.A., Oliveira, G. and Goes-Neto, A. MIMS

Environmental/Metagenome sample from biofilm metagenome. Genbank https://identifiers.org/ncbi/insdc:SAMN02934078 (2017).

211. Vaz, A.B., Fonseca, P.L., Leite, L.R., Badotti, F., Salim, A.C., Araujo, F.M., CuadrosOrellana, S., Duarte, A.A., Rosa, C.A., Oliveira, G. and Goes-Neto, A. MIMS

Environmental/Metagenome sample from biofilm metagenome. Genbank https://identifiers.org/ncbi/insdc:SAMN02934079 (2017).

212. Australian Antarctic Data Center, http://dx.doi.org/10.4225/15/526f42ada05b1 (2016).

213. Hartmann, M., Howes, C.G., VanInsberghe, D., Yu, H., Bachar, D., Christen, R., Nilsson, R.H., Hallam, S.J. and Mohn, W.W. Significant and persistent impact of timber harvesting on soil microbial communities in Northern coniferous forests. Supplementary Data 2

https://staticcontent.springer.com/esm/art\%3A10.1038\%2Fismej.2012.84/MediaObjects/413962012 BF ismej201284 MOESM78 ESM.zip (2012).

214. Rime, T., Hartmann, M. and Frey, B. Potential sources of microbial colonizers in an initial soil ecosystem after retreat of an alpine glacier. Supplementary Information https://staticcontent.springer.com/esm/art\%3A10.1038\%2Fismej.2015.238/MediaObjects/41396 2016 BFismej2015238 MOESM67 ESM.zip (2016).

215. Czech Academy of Sciences, www.biomed.cas.cz/mbu/lbwrf/metastudy datasets/GF0001.zip. 216. Czech Academy of Sciences, www.biomed.cas.cz/mbu/lbwrf/metastudy datasets/GF0002.zip, 217. Czech Academy of Sciences, www.biomed.cas.cz/mbu/lbwrf/metastudy datasets/GF0003.zip, 218. Czech Academy of Sciences, www.biomed.cas.cz/mbu/lbwrf/metastudy datasets/GF0004.zip, 219. Czech Academy of Sciences, www.biomed.cas.cz/mbu/lbwrf/metastudy datasets/GF0005.zip, 220. Czech Academy of Sciences, www.biomed.cas.cz/mbu/lbwrf/metastudy datasets/GF0006.zip, 221. Czech Academy of Sciences, www.biomed.cas.cz/mbu/lbwrf/metastudy datasets/GF0007.zip, 222. Czech Academy of Sciences, www.biomed.cas.cz/mbu/lbwrf/metastudy datasets/GF0008.zip, 223. Czech Academy of Sciences, www.biomed.cas.cz/mbu/lbwrf/metastudy datasets/GF0009.zip, 224. Czech Academy of Sciences, www.biomed.cas.cz/mbu/lbwrf/metastudy datasets/GF0010.zip, 225. Czech Academy of Sciences, www.biomed.cas.cz/mbu/lbwrf/metastudy datasets/GF0011.zip, 226. Czech Academy of Sciences, www.biomed.cas.cz/mbu/lbwrf/metastudy datasets/GF0012.zip. 
bioRxiv preprint doi: https://doi.org/10.1101/2020.04.24.060384; this version posted April 25, 2020. The copyright holder for this preprint (which was not certified by peer review) is the author/funder, who has granted bioRxiv a license to display the preprint in perpetuity. It is made available under aCC-BY-NC-ND 4.0 International license.

227. Anslan, S., Nilsson, R.H., Wurzbacher, C., Baldrian, P., Tedersoo, L. \& Bahram, M. Great differences in performance and outcome of high-throughput sequencing data analysis platforms for fungal metabarcoding. Mycokeys 39, 29-40 (2018). 
bioRxiv preprint doi: https://doi.org/10.1101/2020.04 24.060384; this version posted April 25, 2020. The copyright holder for this preprint (which was not certified by peer review) is the author/funder, who has granted bioRxiv a license to display the preprint in perpetuity. It is made available under aCC-BY-NC-ND 4.0 International license.

\section{Figure Legends}

Figure 1: Map of locations of samples contained in the GlobalFungi database. Each point represents one or several samples where fungal community composition was reported using high-throughput-sequencing methods targeting the ITS1 or ITS2 marker of fungi. The map was created using the 'leaflet' package that uses an open-source JavaScript library for mobile-friendly interactive maps (GNU GENERAL PUBLIC LICENSE).

Figure 2: Processing of raw sequencing data for the GlobalFungi database. Workflow of processing of sequencing data included in the GlobalFungi database.

Figure 3: User interface to access the GlobalFungi database. 


\section{Tables}

Table 1: List of metadata contained in the GlobalFungi database. The table lists identifiers, units and sources of metadata contained in the database with the description of their content. Data source "original paper" may also represent additional metadata provided by the authors of the paper.

\begin{tabular}{|c|c|c|c|}
\hline $\begin{array}{l}\text { Metadata } \\
\text { identifier }\end{array}$ & Unit & Description of content & Source \\
\hline Sample ID & & unique identifier & generated \\
\hline Longitude & degrees & Geographical longitude & $\begin{array}{l}\text { original } \\
\text { paper }\end{array}$ \\
\hline Latitude & degrees & Geographical latitude & $\begin{array}{l}\text { original } \\
\text { paper }\end{array}$ \\
\hline Continent & & $\begin{array}{l}\text { One of the following: } \\
\text { Africa/Antarctica/Asia/Australia/Europe/North } \\
\text { America/South America }\end{array}$ & $\begin{array}{l}\text { original } \\
\text { paper }\end{array}$ \\
\hline $\begin{array}{l}\text { Sample } \\
\text { type } \\
\text { Biome }\end{array}$ & & $\begin{array}{l}\text { One of the following: soil/rhizosphere } \\
\text { soil/litter/litter+humus/deadwood/lichen/shoot/root } \\
\text { One of the following: forest biome/woodland } \\
\text { biome/shrubland biome/grassland biome/desert } \\
\text { biome/tundra biome/mangrove }\end{array}$ & $\begin{array}{l}\text { original } \\
\text { paper }\end{array}$ \\
\hline & & $\begin{array}{l}\text { biome/anthropogenic terrestrial biome/marine } \\
\text { biome/freshwater biome/polar desert biome }\end{array}$ & $\begin{array}{l}\text { original } \\
\text { paper }\end{array}$ \\
\hline $\begin{array}{l}\text { Sampling } \\
\text { year } \\
\text { Primers }\end{array}$ & & Year of sample collection & $\begin{array}{l}\text { original } \\
\text { paper } \\
\text { original }\end{array}$ \\
\hline $\mathrm{pH}$ & & Primers used & $\begin{array}{l}\text { paper } \\
\text { original }\end{array}$ \\
\hline & & $\mathrm{pH}$ & paper \\
\hline ITS total & & Number of full ITS sequences extracted & generated \\
\hline $\operatorname{MAT}\left({ }^{\circ} \mathrm{C}\right)$ & ${ }^{\circ} \mathrm{C}$ & Mean annual temperature from CHELSA database & CHELSA \\
\hline $\operatorname{MAP}(\mathrm{mm})$ & $\mathrm{mm}$ & Mean annual precipitation from CHELSA database & CHELSA \\
\hline
\end{tabular}


Online-only Table 1: List of papers that were used as data sources for GlobalFungi database with digital object identifiers.

\begin{tabular}{|c|c|}
\hline Paper & Doi: \\
\hline $\begin{array}{l}\text { Almario, J., et al.: 2017. Root-associated fungal } \\
\text { microbiota of nonmycorrhizal Arabis alpina and its } \\
\text { contribution to plant phosphorus nutrition. } \\
\text { Proceedings of the National Academy of Sciences of } \\
\text { the United States of America 114(44):E9403-E9412. }\end{array}$ & 10.1073/pnas.1710455114 \\
\hline $\begin{array}{l}\text { Alvarez-Garrido, L., et al.: 2019. Distributional shifts in } \\
\text { ectomycorrizhal fungal communities lag behind } \\
\text { climate-driven tree upward migration in a conifer } \\
\text { forest-high elevation shrubland ecotone. Soil Biology } \\
\text { \& Biochemistry 137:107545. }\end{array}$ & 10.1016/j.soilbio.2019.107545 \\
\hline $\begin{array}{l}\text { Alzarhani, A. K., et al.: } 2019 \text {. Are drivers of root- } \\
\text { associated fungal community structure context } \\
\text { specific?. ISME Journal 13(5):1330-1344. }\end{array}$ & $10.1038 / s 41396-019-0350-y$ \\
\hline $\begin{array}{l}\text { Anthony, M. A., et al.: } 2017 \text {. Fungal community } \\
\text { homogenization, shift in dominant trophic guild, and } \\
\text { appearance of novel taxa with biotic invasion. } \\
\text { Ecosphere 8(9):e01951. }\end{array}$ & 10.1002/ecs2.1951 \\
\hline $\begin{array}{l}\text { Bahram, M., et al.: } 2016 \text {. Stochastic distribution of } \\
\text { small soil eukaryotes resulting from high dispersal and } \\
\text { drift in a local environment. ISME Journal 10(4):885- } \\
896 .\end{array}$ & 10.1038/ismej.2015.164 \\
\hline $\begin{array}{l}\text { Bach, E. M., et al.: } 2018 \text {. Greatest soil microbial } \\
\text { diversity found in micro-habitats. Soil Biology \& } \\
\text { Biochemistry 118:217-226. }\end{array}$ & 10.1016/j.soilbio.2017.12.018 \\
\hline $\begin{array}{l}\text { Baldrian, P., et al.: } 2012 \text {. Active and total microbial } \\
\text { communities in forest soil are largely different and } \\
\text { highly stratified during decomposition. ISME Journal } \\
6(2): 248-258 \text {. }\end{array}$ & 10.1038/ismej.2011.95 \\
\hline $\begin{array}{l}\text { Baldrian, P., et al.: } 2016 \text {. Fungi associated with } \\
\text { decomposing deadwood in a natural beech- } \\
\text { dominated forest. Fungal Ecology 23:109-122. }\end{array}$ & 10.1016/j.funeco.2016.07.001 \\
\hline $\begin{array}{l}\text { Barnes, C. J., et al.: 2016. Unexpectedly High Beta- } \\
\text { Diversity of Root-Associated Fungal Communities in } \\
\text { the Bolivian Andes. Frontiers in Microbiology 7:1377. }\end{array}$ & 10.3389/fmicb.2016.01377 \\
\hline $\begin{array}{l}\text { Bergottini, V. M., et al.: 2017. Exploring the diversity } \\
\text { of the root-associated microbiome of Ilex } \\
\text { paraguariensis St. Hil. (Yerba Mate). Applied Soil } \\
\text { Ecology 109:23-31. }\end{array}$ & 10.1016/j.apsoil.2016.09.013 \\
\hline $\begin{array}{l}\text { Bickford, W. A., et al.: } 2018 \text {. Root endophytes and } \\
\text { invasiveness: no difference between native and non- } \\
\text { native Phragmites in the Great Lakes Region. } \\
\text { Ecosphere } 9(12): \text { e02526. }\end{array}$ & $10.1002 /$ ecs 2.2526 \\
\hline $\begin{array}{l}\text { Bissett, A., et al.: 2016. Introducing BASE: the Biomes } \\
\text { of Australian Soil Environments soil microbial diversity } \\
\text { database. GigaScience 5(1):21. }\end{array}$ & $10.1186 / s 13742-016-0126-5$ \\
\hline $\begin{array}{l}\text { Buee, M., et al.: 2009. } 454 \text { Pyrosequencing analyses of } \\
\text { forest soils reveal an unexpectedly high fungal } \\
\text { diversity. New Phytologist 184(2):449-456. }\end{array}$ & $\begin{array}{l}\text { 10.1111/j.1469- } \\
8137.2009 .03003 . x\end{array}$ \\
\hline
\end{tabular}


Clemmensen, K. E., et al.: 2015. Carbon sequestration is related to mycorrhizal fungal community shifts during long-term succession in boreal forests. New Phytologist 205(4):1525-1536.

Cline, L. C., et al.: 2018. Ecological and functional effects of fungal endophytes on wood decomposition. Functional Ecology 32(1):181-191.

Coleine, C., et al.: 2018. Antarctic cryptoendolithic fungal communities are highly adapted and dominated by Lecanoromycetes and Dothideomycetes. Frontiers in Microbiology 9:1392.

Cordier, T., et al.: 2012. Spatial variability of phyllosphere fungal assemblages: genetic distance predominates over geographic distance in a European beech stand (Fagus syluatica). Fungal Ecology 5:509-

520.

Cox, F., et al.: 2016. Not poles apart: Antarctic soil fungal communities show similarities to those of the distant Arctic. Ecology Letters 19(5):528-536.

Cross, H., et al.: 2017. Fungal diversity and seasonal succession in ash leaves infected by the invasive ascomycete Hymenoscyphus fraxineus. New Phytologist 213(3):1405-1417.

Davey, M. L., et al.: 2012. Seasonal trends in the biomass and structure of bryophyte-associated fungal communities explored by 454 pyrosequencing. New Phytologist 195(4):844-856.

Davey, M. L., et al.: 2013. Amplicon-pyrosequencingbased detection of compositional shifts in bryophyteassociated fungal communities along an elevation gradient. Molecular Ecology 22(2):368-383. Davey, M. L., et al.: 2014. Forestry impacts on the hidden fungal biodiversity associated with bryophytes. FEMS Microbiology Ecology 90(1):313325.

De Beeck, M. O., et al.: 2014. Comparison and Validation of Some ITS Primer Pairs Useful for Fungal Metabarcoding Studies. PLoS One 9(6):e97629. Dean, S. L., et al.: 2017. A study of Glycine max (soybean) fungal communities under different agricultural practices. Plant Gene 11:8-16.

DeBeeck, M. O., et al.: 2015. Impact of metal pollution on fungal diversity and community structures. Environmental Microbiology 17(6):2035-2047. Delgado-Baquerizo, M., et al.: 2017. Soil microbial communities drive the resistance of ecosystem multifunctionality to global change in drylands across the globe. Ecology Letters 20(10):1295-1305. Elliott, D. R., et al.: 2015. Bacterial and Fungal Communities in a Degraded Ombrotrophic Peatland Undergoing Natural and Managed Re-Vegetation. PLoS One 10(5):e0124726. 10.1111/nph.13208

$10.1111 / 1365-2435.12949$

10.3389/fmicb.2018.01392

10.1016/j.funeco.2011.12.004

10.1111/ele. 12587

10.1111/nph.14204

10.1111/j.1469-

8137.2012.04215.x

10.1111/mec.12122

10.1111/1574-6941.12386

10.1371/journal.pone.0097629

10.1016/j.plgene.2016.11.003

$10.1111 / 1462-2920.12547$

10.1111/ele.12826

10.1371/journal.pone.0124726 
Fan, K., et al.: 2018. Wheat rhizosphere harbors a less complex and more stable microbial co-occurrence pattern than bulk soil.. Soil Biology \& Biochemistry 125:251-260.

Fernandez, C. W., et al.: 2017. Ectomycorrhizal fungal response to warming is linked to poor host performance at the boreal-temperate ecotone. Global Change Biology 23(4):1598-1609.

Fernandez-Martinez, M. A., et al.: 2017. Microbial succession dynamics along glacier forefield chronosequences in Tierra del Fuego (Chile). Polar Biology 40(10):1939-1957.

Fernandez-Mendoza, F., et al.: 2017. ITS1 metabarcoding highlights low specificity of lichen mycobiomes at a local scale. Molecular Ecology 26(18):4811-4830.

Frey, B., et al.: 2016. Microbial diversity in European alpine permafrost and active layers. FEMS Microbiology Ecology 92(3):fiw018.

Fukasawa, Y. and Matsuoka, S.: 2015. Communities of wood-inhabiting fungi in dead pine logs along a geographical gradient in Japan. Fungal Ecology 18:7582.

Galazka, A. and Grzadziel, J.: 2018. Fungal Genetics and Functional Diversity of Microbial Communities in the Soil under Long-Term Monoculture of Maize Using Different Cultivation Techniques.. Frontiers in Microbiology 9:76.

Gannes, V., et al.: 2016. Microbial Community Structure and Function of Soil Following Ecosystem Conversion from Native Forests to Teak Plantation Forests. Frontiers in Microbiology 7:1976.

Gao, C., et al.: 2015. Community assembly of ectomycorrhizal fungi along a subtropical secondary forest succession. New Phytologist 205(2):771-785.

Ge, Z. W., et al.: 2017. Soil pH and mineral nutrients strongly influence truffles and other ectomycorrhizal fungi associated with commercial pecans (Carya illinoinensis). Plant and Soil 418(1-2):493-505.

Gehring, C. A., et al.: 2016. Cheatgrass invasion alters the abundance and composition of dark septate fungal communities in sagebrush steppe. Botany 94(6):481-491.

Geml, J., et al.: 2014. The Contribution of DNA Metabarcoding to Fungal Conservation: Diversity Assessment, Habitat Partitioning and Mapping RedListed Fungi in Protected Coastal Salix repens Communities in the Netherlands. PLoS One 9(6):e99852.

Geml, J., et al.: 2015. Long-term warming alters richness and composition of taxonomic and functional 10.1016/j.soilbio.2018.07.022

10.1111/gcb.13510

10.1007/s00300-017-2110-7

10.1111/mec.14244

10.1093/femsec/fiw018

10.1016/j.funeco.2015.09.008

10.3389/fmicb.2018.00076

10.3389/fmicb.2016.01976

10.1111/nph.13068

10.1007/s11104-017-3312-z

10.1139/cjb-2015-0237

10.1371/journal.pone.0099852

10.1093/femsec/fiv095 
groups of arctic fungi. FEMS Microbiology Ecology 91(8):fiv095.

George, P. B., et al.: 2019. Divergent national-scale trends of microbial and animal biodiversity revealed across diverse temperate soil ecosystems.. Nature Communications 10:1107.

Glassman, S. I., et al.: 2016. Ectomycorrhizal fungal spore bank recovery after a severe forest fire: some like it hot. ISME Journal 10(5):1228-1239.

Glassman, S. I., et al.: 2017. Environmental filtering by $\mathrm{pH}$ and soil nutrients drives community assembly in fungi at fine spatial scales. Molecular Ecology 26(24):6960-6973.

Glynou, K., et al.: 2018. Facultative root-colonizing fungi dominate endophytic assemblages in roots of nonmycorrhizal Microthlaspi species. New Phytologist 217(3):1190-1202.

Goldmann, K., et al.: 2015. Forest Management Type Influences Diversity and Community Composition of Soil Fungi across Temperate Forest Ecosystems. Frontiers in Microbiology 6:1300.

Goldmann, K., et al.: 2016. Divergent habitat filtering of root and soil fungal communities in temperate beech forests. Scientific Reports 11:31439.

Gomes, S. I. F., et al.: 2017. Arbuscular mycorrhizal interactions of mycoheterotrophic Thismia are more specialized than in autotrophic plants. New Phytologist 213(3):1418-1427.

Gomes, S. I. F., et al.: 2017. Fungal-host diversity among mycoheterotrophic plants increases proportionally to their fungal-host overlap. Ecology and Evolution 7(10):3623-3630.

Gourmelon, V., et al.: 2016. Environmental and Geographical Factors Structure Soil Microbial Diversity in New Caledonian Ultramafic Substrates: A Metagenomic Approach. PLoS One 11(12):e0167405. Grau, O., et al.: 2017. Abrupt changes in the composition and function of fungal communities along an environmental gradient in the high Arctic. Molecular Ecology 26(18):4798-4810.

Guo, J. et al.: 2019. Soil fungal assemblage complexity is dependent on soil fertility and dominated by deterministic processes. New Phytologist 226(1):232243.

Harrison, J. G., et al.: 2016. Vertical stratification of the foliar fungal community in the world's tallest trees. American Journal of Botany 103(12):2087-2095. Hartmann, M., et al.: 2012. Significant and persistent impact of timber harvesting on soil microbial communities in Northern coniferous forests. ISME Journal 6(12):2199-2218.
$10.1038 / \mathrm{s} 41467-019-09031-1$

10.1038/ismej.2015.182

10.1111/mec.14414

10.1111/nph.14873

10.3389/fmicb.2015.01300

10.1038/srep31439

10.1111/nph.14249

10.1002/ece3.2974

10.1371/journal.pone.0167405

10.1111/mec.14227

10.1111/nph.16345

10.3732/ajb.1600277

10.1038/ismej.2012.84 
bioRxiv preprint doi: https://doi.org/10.1101/2020.04 24.060384 this version posted April 25, 2020. The copyright holder for this preprint (which was not certified by peer review) is the author/funder, who has granted bioRxiv a license to display the preprint in perpetuity. It is made available under aCC-BY-NC-ND 4.0 International license.

He, D., et al.: 2017. Diversity and co-occurrence network of soil fungi are more responsive than those of bacteria to shifts in precipitation seasonality in a subtropical forest. Soil Biology \& Biochemistry 115:499-510.

He, F., et al.: 2016. Changes in composition and diversity of fungal communities along Quercus mongolica forests developments in Northeast China. Applied Soil Ecology 100:162-171.

Hiiesalu, I., et al.: 2017. Plant species richness and productivity determine the diversity of soil fungal guilds in temperate coniferous forest and bog habitats. Molecular Ecology 26(18):4846-4858. Hoppe, B., et al.: 2015. Linking molecular deadwoodinhabiting fungal diversity and community dynamics to ecosystem functions and processes in Central European forests. Fungal Diversity 77(1):367-379. Chaput, D. L., et al.: 2015. Profiling Microbial Communities in Manganese Remediation Systems Treating Coal Mine Drainage. Applied and Environmental Microbiology 81(6):2189-2198. Ihrmark, K., et al.: 2012. New primers to amplify the fungal ITS2 region - evaluation by 454-sequencing of artificial and natural communities. FEMS Microbiology Ecology 82(3):666-677.

Izuno, A., et al.: 2016. Structure of phyllosphere fungal communities in a tropical dipterocarp plantation: A massively parallel next-generation sequencing analysis. Mycoscience 57(3):171-180. Izuno, A., et al.: 2016. Vertical Structure of Phyllosphere Fungal Communities in a Tropical Forest in Thailand Uncovered by High-Throughput Sequencing. PLoS One 11(11):e0166669. Jarvis, S. G., et al.: 2015. Strong altitudinal partitioning in the distributions of ectomycorrhizal fungi along a short (300 m) elevation gradient. New Phytologist 206(3):1145-1155.

Ji, M., et al.: 2016. Microbial diversity at Mitchell Peninsula, Eastern Antarctica: a potential biodiversity "hotspot". Polar Biology 39(2):237-249.

Jiao, S., et al.: 2018. Soil microbiomes with distinct assemblies through vertical soil profiles drive the cycling of multiple nutrients in reforested ecosystems. Microbiome 6(1):146.

Johansen, R. B., et al.: 2016. A native and an invasive dune grass share similar, patchily distributed, rootassociated fungal communities. Fungal Ecology 23:141-155.

Jumpponen, A. and Jones, K. L.: 2010. Seasonally dynamic fungal communities in the Quercus macrocarpa phyllosphere differ between urban and 10.1016/j.soilbio.2017.09.023

10.1016/j.apsoil.2015.12.014

10.1111/mec.14246

$10.1007 / \mathrm{s} 13225-015-0341-x$

10.1128/AEM.03643-14

10.1111/j.1574-

6941.2012.01437.x

10.1016/j.myc.2015.12.005

10.1371/journal.pone.0166669

10.1111/nph.13315

10.1007/s00300-015-1776-y

10.1186/s40168-018-0526-0

10.1016/j.funeco.2016.08.003

10.1111/j.1469-

8137.2010.03197.x 
nonurban environments. New Phytologist 186(2):496-

513.

Jumpponen, A., et al.: 2010. Massively parallel 454-

sequencing of fungal communities in Quercus spp

ectomycorrhizas indicates seasonal dynamics in urban

and rural sites. Molecular Ecology 19(1):41-53.

Kadowaki, K., et al.: 2014. Detection of the horizontal

spatial structure of soil fungal communities in a natural forest. Population Ecology 56(2):301-310.

Kamutando, C. N., et al.: 2017. Soil nutritional status and biogeography influence rhizosphere microbial communities associated with the invasive tree Acacia dealbata. Scientific Reports 7:6472.

Kazartsev, l., et al.: 2018. Decaying Picea abies log bark hosts diverse fungal communities. Fungal Ecology 33:1-12.

Kerfahi, D., et al.: 2016. Rainforest Conversion to Rubber Plantation May Not Result in Lower Soil Diversity of Bacteria, Fungi, and Nematodes.

Microbial Ecology 72(2):359-371.

Kielak, A. M., et al.: 2016. Bacterial Community

Succession in Pine-Wood Decomposition. Frontiers in Microbiology 7:231.

Kolarikova, Z., et al.: 2017. Root-associated fungal communities along a primary succession on a mine spoil: Distinct ecological guilds assemble differently. Soil Biology \& Biochemistry 113:143-152.

Kyaschenko, J., et al.: 2017. Shift in fungal communities and associated enzyme activities along an age gradient of managed Pinus sylvestris stands. ISME Journal 11(4):863-874.

Lanzen, A., et al.: 2016. Multi-targeted metagenetic analysis of the influence of climate and environmental parameters on soil microbial communities along an elevational gradient. Scientific Reports 6:28257. Leff, J. W., et al.: 2015. Consistent responses of soil microbial communities to elevated nutrient inputs in grasslands across the globe. Proceedings of the National Academy of Sciences of the United States of America 112(35):10967-10972.

Li, Y., et al.: 2016. Changes of soil microbial community under different degraded gradients of alpine meadow. Agriculture, Ecosystems and Environment 222:213-222.

Liu, C., et al.: 2016. The influence of soil properties on the size and structure of bacterial and fungal communities along a paddy soil chronosequence. European Journal of Soil Biology 76:9-18.

Liu, J., et al.: 2015. Soil carbon content drives the biogeographical distribution of fungal communities in the black soil zone of northeast China. Soil Biology \& Biochemistry 83:29-39.
$10.1111 / \mathrm{j} .1365-$

294X.2009.04483.x

10.1007/s10144-013-0424-z

10.1038/s41598-017-07018-w

10.1016/j.funeco.2017.12.005

$10.1007 / \mathrm{s} 00248-016-0790-0$

10.3389/fmicb.2016.00231

10.1016/j.soilbio.2017.06.004

10.1038/ismej.2016.184

10.1038/srep28257

10.1073/pnas.1508382112

10.1016/j.agee.2016.02.020

10.1016/j.ejsobi.2016.06.002

10.1016/j.soilbio.2015.01.009 
Marin, C., et al.: 2017. Functional land-use change effects on soil fungal communities in Chilean temperate rainforests. Journal of Soil Science and Plant Nutrition 17(4):985-1002.

Martirosyan, V., et al.: 2016. Desert Perennial Shrubs Shape the Microbial-Community Miscellany in Laimosphere and Phyllosphere Space. Microbial Ecology 72(3):659-668.

Matsuoka, S., et al.: 2016. Disentangling the relative importance of host tree community, abiotic environment and spatial factors on ectomycorrhizal fungal assemblages along an elevation gradient. FEMS Microbiology Ecology 92(5):fiw044.

Matsuoka, S., et al.: 2016. Temporal distance decay of similarity of ectomycorrhizal fungal community composition in a subtropical evergreen forest in Japan. FEMS Microbiology Ecology 92(5):fiw061.

Matsuoka, S., et al.: 2018. Taxonomic, functional, and phylogenetic diversity of fungi along primary successional and elevational gradients near Mount Robson, British Columbia. Polar Science 21:165-171. McHugh, T. A. and Schwartz, E.: 2015. Changes in plant community composition and reduced precipitation have limited effects on the structure of soil bacterial and fungal communities present in a semiarid grassland. Plant and Soil 388(1-2):175-186. McHugh, T. A., et al.: 2014. Minor Changes in Soil Bacterial and Fungal Community Composition Occur in Response to Monsoon Precipitation in a Semiarid Grassland. Microbial Ecology 68(2):370-378. Mello, A., et al.: 2011. ITS-1 versus ITS-2 pyrosequencing: a comparison of fungal populations in truffle grounds. Mycologia 103(6):1184-1193. Mendoza, J. R., et al.: 2017. Understanding the mycobiota of maize from the highlands of Guatemala, and implications for maize quality and safety. Crop Protection 101:5-11.

Mirmajlessi, S. M., et al.: 2018. Survey of Soil Fungal Communities in Strawberry Fields by Illumina Amplicon Sequencing.. Eurasian Soil Science 51(6):682-691.

Miura, T., et al.: 2017. Is microbial terroir related to geographic distance between vineyards?.

Environmental Microbiology Reports 9(6):742-749. Montagna, M., et al.: 2018. Differential biodiversity responses between kingdoms (plants, fungi, bacteria and metazoa) along an Alpine succession gradient. Molecular Ecology 27(18):3671-3685.

Moussa, T. A. A., et al.: 2017. Comparative metagenomics approaches to characterize the soil fungal communities of western coastal region, Saudi Arabia. PLoS One 12(9):e0185096.
10.4067/S0718-

95162017000400011

$10.1007 / \mathrm{s} 00248-016-0822-9$

10.1093/femsec/fiw044

10.1093/femsec/fiw061

10.1016/j.polar.2018.09.004

10.1007/s11104-014-2269-4

$10.1007 / \mathrm{s} 00248-014-0416-3$

$10.3852 / 11-027$

10.1016/j.cropro.2017.07.009

10.1134/S106422931806011X

10.1111/1758-2229.12589

10.1111/mec.14817

10.1371/journal.pone.0185096 
Muller, L. A. H. and Hilger, H. H.: 2015. Insights into the effects of serpentine soil conditions on the community composition of fungal symbionts in the roots of Onosma echioides. Soil Biology \& Biochemistry 81:1-8.

Mundra, S., et al.: 2015. Arctic fungal communities associated with roots of Bistorta vivipara do not respond to the same fine-scale edaphic gradients as the aboveground vegetation. New Phytologist 205(4):1587-1597.

Nacke, H., et al.: 2016. Fine Spatial Scale Variation of Soil Microbial Communities under European Beech and Norway Spruce. Frontiers in Microbiology 7:2067. Newsham, K. K., et al.: 2016. Relationship between soil fungal diversity and temperature in the maritime Antarctic. Nature Climate Change 6(2):182.

Nguyen, D., et al.: 2016. Do foliar fungal communities of Norway spruce shift along a tree species diversity gradient in mature European forests?. Fungal Ecology 23:97-108.

Nguyen, D., et al.: 2017. Foliar fungi of Betula pendula: impact of tree species mixtures and assessment methods. Scientific Reports 7:41801.

Oh, S.-Y., et al.: 2016. Distinctive Feature of Microbial Communities and Bacterial Functional Profiles in Tricholoma matsutake Dominant Soil. PLoS One 11(12):e0168573.

Oja, J., et al.: 2015. Temporal patterns of orchid mycorrhizal fungi in meadows and forests as revealed by 454 pyrosequencing. New Phytologist 205(4):16081618.

Oja, J., et al.: 2017. Local-scale spatial structure and community composition of orchid mycorrhizal fungi in semi-natural grasslands. Mycorrhiza 27(4):355-367.

Oono, R., et al.: 2017. Distance decay relationships in foliar fungal endophytes are driven by rare taxa.

Environmental Microbiology 19(7):2794-2805.

Oono, R.: 2017. A confidence interval analysis of sampling effort, sequencing depth, and taxonomic resolution of fungal community ecology in the era of high-throughput sequencing. PLoS One 12(12):e0189796.

Ovaskainen, O., et al.: 2010. Identifying woodinhabiting fungi with 454 sequencing - what is the probability that BLAST gives the correct species?. Fungal Ecology 3:274-283.

Park, M. S., et al.: 2018. Diversity of fungi associated with roots of Calanthe orchid species in Korea. Journal of Microbiology 56(1):49-55.

Peay, K. G., et al.: 2013. Strong coupling of plant and fungal community structure across western Amazonian rainforests. ISME Journal 7(9):1852-1861. 10.1016/j.soilbio.2014.10.027

10.1111/nph.13216

10.3389/fmicb.2016.02067

10.1038/NCLIMATE2806

10.1016/j.funeco.2016.07.003

10.1038/srep41801

10.1371/journal.pone.0168573

10.1111/nph.13223

$10.1007 / \mathrm{s} 00572-016-0755-7$

10.1111/1462-2920.1379

10.1371/journal.pone.0189796

10.1016/j.funeco.2010.01.001

10.1007/s12275-018-7319-9

10.1038/ismej.2013.66 
Peay, K. G., et al.: 2017. Convergence and contrast in the community structure of Bacteria, Fungi and Archaea along a tropical elevation-climate gradient. FEMS Microbiology Ecology 93(5):fix045.

Persoh, D.: 2013. Factors shaping community structure of endophytic fungi-evidence from the Pinus-Viscum-system. Fungal Diversity 60(1):55-69. Polackova, T., et al.: 2016. Drivers of yeast community composition in the litter and soil of a temperate forest. FEMS Microbiology Ecology 93(2):fiw223. Poosakkannu, A., et al.: 2017. Microbial community composition but not diversity changes along succession in arctic sand dunes. Environmental Microbiology 19(2):698-709.

Porter, T. M., et al.: 2016. Ribosomal DNA and Plastid Markers Used to Sample Fungal and Plant Communities from Wetland Soils Reveals Complementary Biotas. PLoS One 11(1):e0142759. Purahong, W., et al.: 2017. Characterization of Unexplored Deadwood Mycobiome in Highly Diverse Subtropical Forests Using Culture-independent Molecular Technique. Frontiers in Microbiology 8:574. Purahong, W., et al.: 2018. Determinants of Deadwood-Inhabiting Fungal Communities in Temperate Forests: Molecular Evidence From a Large Scale Deadwood Decomposition Experiment. Frontiers in Microbiology 9:2120.

Purahong, W., et al.: 2018. Increasing N deposition impacts neither diversity nor functions of deadwoodinhabiting fungal communities, but adaptation and functional redundancy ensure ecosystem function. Environmental Microbiology 20(5):1693-1710. Purahong, W., et al.: 2019. Characterization of the Castanopsis carlesii deadwood mycobiome by Pacbio sequencing of the full-length fungal nuclear ribosomal internal transcribed spacer (ITS).. Frontiers in Microbiology 10:983.

Rajala, T., et al.: 2015. Habitat models of woodinhabiting fungi along a decay gradient of Norway spruce logs. Fungal Ecology 18:48-55.

Ramirez, K. S., et al.: 2019. Range-expansion effects on the belowground plant microbiome. Nature Ecology and Evolution 3(4):604.

Ramona, M.,et al.: 2018. Rhizosheath microbial community assembly of sympatric desert speargrasses is independent of the plant host. Microbiome 6(1):215.

Rasmussen, P.U., et al.: 2018. Multiscale patterns and drivers of arbuscular mycorrhizal fungal communities in the roots and root-associated soil of a wild perennial herb. New Phytologist 220(4):1248-1261 10.1093/femsec/fix045

$10.1007 / \mathrm{s} 13225-013-0225-\mathrm{x}$

10.1093/femsec/fiw223

$10.1111 / 1462-2920.13599$

10.1371/journal.pone.0142759

10.3389/fmicb.2017.00574

10.3389/fmicb.2018.02120

$10.1111 / 1462-2920.14081$

10.3389/fmicb.2019.00983

10.1016/j.funeco.2015.08.007

10.1038/s41559-019-0828-z

10.1186/s40168-018-0597-y

10.1111/nph.15088 
Reese, A. T., et al.: 2016. Urban stress is associated with variation in microbial species composition-but not richness-in Manhattan. ISME Journal 10(3):751760.

Ren, C., et al.: 2018. Differential responses of soil microbial biomass, diversity, and compositions to altitudinal gradients depend on plant and soil characteristics. Science of the Total Environment 610:750-758.

Rime, T., et al.: 2015. Vertical distribution of the soil microbiota along a successional gradient in a glacier forefield. Molecular Ecology 24(5):1091-1108.

Rime, T., et al.: 2016. Potential sources of microbial colonizers in an initial soil ecosystem after retreat of an alpine glacier. ISME Journal 10(7):1625-1641. Rincon, A., et al.: 2015. Compartmentalized and contrasted response of ectomycorrhizal and soil fungal communities of Scots pine forests along elevation gradients in France and Spain.

Environmental Microbiology 17(8):3009-3024.

Rogers, T. J., et al.: 2018. Exploring variation in phyllosphere microbial communities across four hemlock species.. Ecosphere 9(12):e02524.

Roy-Bolduc, A., et al.: 2016. High richness of ectomycorrhizal fungi and low host specificity in a coastal sand dune ecosystem revealed by network analysis. Ecology and Evolution 6(1):349-362.

Roy-Bolduc, A., et al.: 2016. Strong linkage between plant and soil fungal communities along a successional coastal dune system. FEMS Microbiology Ecology 92(10):fiw156.

Saitta, A., et al.: 2018. Tree species identity and diversity drive fungal richness and community composition along an elevational gradient in a Mediterranean ecosystem. Mycorrhiza 28(1):39-47. Santalahti, M., et al.: 2016. Vertical and seasonal dynamics of fungal communities in boreal Scots pine forest soil. FEMS Microbiology Ecology 92(11):fiw170. Santalahti, M., et al.: 2018. Reindeer grazing alter soil fungal community structure and litter decomposition related enzyme activities in boreal coniferous forests in Finnish Lapland. Applied Soil Ecology 132:74-82. Sapkota, R. and Nicolaisen, M.: 2018. Cropping history shapes fungal, oomycete and nematode communities in arable soils and affects cavity spot in carrot. Agriculture, Ecosystems and Environment 257:120-

131.

Saravesi, K., et al.: 2015. Moth Outbreaks Alter RootAssociated Fungal Communities in Subarctic Mountain Birch Forests. Microbial Ecology 69(4):788-797.

Semenova, T. A., et al.: 2016. Compositional and functional shifts in arctic fungal communities in 10.3389/fmicb.2017.00574

10.1016/j.scitotenv.2017.08.110

10.1111/mec.13051

10.1038/ismej.2015.238

10.1111/1462-2920.12894

$10.1002 /$ ecs 2.2524

10.1002/ece3.1881

10.1093/femsec/fiw156

$10.1007 / \mathrm{s} 00572-017-0806-8$

10.1093/femsec/fiw170

10.1016/j.apsoil.2018.08.013

10.1016/j.agee.2018.01.032

$10.1007 / \mathrm{s} 00248-015-0577-8$

10.1016/j.soilbio.2016.06.001 
response to experimentally increased snow depth.

Soil Biology \& Biochemistry 100:201-209.

Semenova-Nelsen, T. A., et al.: 2019. Frequent fire reorganizes fungal communities and slows decomposition across a heterogeneous pine savanna landscape. New Phytologist 224(2):916-927.

Sharma-Poudyal, D., et al.: 2017. Long-term no-till: A major driver of fungal communities in dryland wheat cropping systems. PLoS One 12(9):e0184611.

Shen, Z., et al.: 2017. Banana Fusarium Wilt Disease Incidence Is Influenced by Shifts of Soil Microbial Communities Under Different Monoculture Spans. Microbial Ecology 75(3):739-750.

Shigyo, N., et al.: 2019. Seasonal Dynamics of Soil Fungal and Bacterial Communities in Cool-Temperate Montane Forests. Frontiers in Microbiology 10:1944. Schappe, T., et al.: 2017. The role of soil chemistry and plant neighbourhoods in structuring fungal communities in three Panamanian rainforests. Journal of Ecology 105(3):569-579.

Schlatter, D. C., et al.: 2018. Fungal community composition and diversity vary with soil depth and landscape position in a no-till wheat-based cropping system. FEMS Microbiology Ecology 94(7):fiy098.

Schlegel, M., et al.: 2018. The Endophytic Mycobiome of European Ash and Sycamore Maple Leaves Geographic Patterns, Host Specificity and Influence of Ash Dieback. Frontiers in Microbiology 9:2345.

Schmidt, P-A., et al.: 2013. Illumina metabarcoding of a soil fungal community. Soil Biology \& Biochemistry 65:128-132.

Schneider-Maunoury, L., et al.: 2018. Is Tuber melanosporum colonizing the roots of herbaceous, non-ectomycorrhizal plants?. Fungal Ecology 31:59-

68.

Schon, M. E., et al.: 2018. Belowground fungal community diversity and composition associated with Norway spruce along an altitudinal gradient. PLoS One 13(12):e0208493.

Schroeder, J. W., et al.: 2018. Community composition and diversity of Neotropical root-associated fungi in common and rare trees. Biotropica 50(4):694-703.

Si, P., et al.: 2018. Rhizosphere Microenvironments of Eight Common Deciduous Fruit Trees Were Shaped by Microbes in Northern China. Frontiers in Microbiology 9:3147.

Siles, J. A. and Margesin, R.: 2016. Abundance and Diversity of Bacterial, Archaeal, and Fungal Communities Along an Altitudinal Gradient in Alpine Forest Soils: What Are the Driving Factors?. Microbial Ecology 72(1):207-220. 10.1111/nph.16096

10.1371/journal.pone.0184611

10.1007/s00248-017-1052-5

10.3389/fmicb.2019.01944

$10.1111 / 1365-2745.12752$

10.1093/femsec/fiy098

10.3389/fmicb.2018.02345

10.1016/j.soilbio.2013.05.014

10.1016/j.funeco.2017.10.004

10.1371/journal.pone.0208493

10.1111/btp.12553

10.3389/fmicb.2018.03147

$10.1007 / \mathrm{s} 00248-016-0748-2$ 
microbial responses are limited to changes in functionality at two Alpine forest sites differing in altitude and vegetation. Scientific Reports 7:2204. Smith, M. E., et al.: 2017. Investigating niche partitioning of ectomycorrhizal fungi in specialized rooting zones of the monodominant leguminous tree Dicymbe corymbosa. New Phytologist 215(1):443-453. Song, H., et al.: 2019. Tropical forest conversion to rubber plantation in southwest China results in lower fungal beta diversity and reduced network complexity. FEMS Microbiology Ecology 95(7):fiz092. Sterkenburg, E., et al.: 2015. Changes in fungal communities along a boreal forest soil fertility gradient. New Phytologist 207(4):1145-1158. Stursova, M., et al.: 2016. Small-scale spatial heterogeneity of ecosystem properties, microbial community composition and microbial activities in a temperate mountain forest soil. FEMS Microbiology Ecology 92(12):fiw185.

Sun, H., et al.: 2015. Fungal Community Shifts in Structure and Function across a Boreal Forest Fire Chronosequence. Applied and Environmental Microbiology 81(22):7869-7880.

Sun, R., et al.: 2018. Tillage Changes Vertical Distribution of Soil Bacterial and Fungal Communities. Frontiers in Microbiology 9:699.

Talbot, J. M., et al.: 2014. Endemism and functional convergence across the North American soil mycobiome. Proceedings of the National Academy of Sciences of the United States of America 111(17): 6341-6346.

Tedersoo, L., et al.: 2010. 454 Pyrosequencing and Sanger sequencing of tropical mycorrhizal fungi provide similar results but reveal substantial methodological biases. New Phytologist 188(1):291301.

Tedersoo, L., et al.: 2014. Global diversity and geography of soil fungi. Science 346(6213):1256688. Tedersoo, L., et al.: 2015. Shotgun metagenomes and multiple primer pair-barcode combinations of amplicons reveal biases in metabarcoding analyses of fungi. Mycokeys 10:1-43.

Tedersoo, L., et al.: 2016. Tree diversity and species identity effects on soil fungi, protists and animals are context dependent. ISME Journal 10(2):346-362.

Thiem, D., et al.: 2017. Ectomycorrhizal and endophytic fungi associated with Alnus glutinosa growing in a saline area of central Poland. Symbiosis 75(1):17-28.

Tian, H., et al.: 2017. Changes in soil microbial communities after 10 years of winter wheat 10.1111/nph.14570

10.1093/femsec/fiz092

10.1111/nph.13426

10.1093/femsec/fiw185

10.1128/AEM.02063-15

10.3389/fmicb.2018.00699

$10.1073 /$ pnas. 1402584111

10.1111/j.1469-

8137.2010.03373.x

10.1126/science. 1256688

10.3897/mycokeys.10.4852

10.1038/ismej.2015.116

10.1007/s13199-017-0512-5

10.1371/journal.pone.0184223 
cultivation versus fallow in an organic-poor soil in the Loess Plateau of China. PLoS One 12(9):e0184223. Tian, J. Q., et al.: 2017. Ecological Succession Pattern of Fungal Community in Soil along a Retreating Glacier. Frontiers in Microbiology 8:1028.

Tian, J. Q., et al.: 2017. Patterns and drivers of fungal diversity along an altitudinal gradient on Mount Gongga, China. Journal of Soils and Sediments 17(12):2856-2865.

Toju, H., et al.: 2016. Ericaceous plant-fungus network in a harsh alpine-subalpine environment. Molecular Ecology 25(13):3242-3257.

Toju, H., et al.: 2016. Network modules and hubs in plant-root fungal biomes. Journal of the Royal Society, Interface 13(116):20151097.

Toole, D. R., et al.: 2017. Differences in soil fungal assemblages associated with native and non-native tree species of varying weediness. Biological Invasions 20(4):891-904.

Truong, C., et al.: 2019. Ectomycorrhizal fungi and soil enzymes exhibit contrasting patterns along elevation gradients in southern Patagonia. New Phytologist 222(4):1936-1950.

Tu, B., et al.: 2017. Microbial diversity in Chinese temperate steppe: unveiling the most influential environmental drivers. FEMS Microbiology Ecology 93(4):fix031.

Urbanova, M., et al.: 2015. Composition of fungal and bacterial communities in forest litter and soil is largely determined by dominant trees. Soil Biology \& Biochemistry 84:53-64.

Urbina, H., et al.: 2016. DNA-metabarcoding uncovers the diversity of soil-inhabiting fungi in the tropical island of Puerto Rico. Mycoscience 57(3):217-227. Uroz, S., et al.: 2016. Specific impacts of beech and Norway spruce on the structure and diversity of the rhizosphere and soil microbial communities. Scientific Reports 6:27756.

Valverde, A., et al.: 2016. Specific Microbial Communities Associate with the Rhizosphere of Welwitschia mirabilis, a Living Fossil. PLoS One 11(4):e0153353.

van der Wal, A., et al.: 2015. Neglected role of fungal community composition in explaining variation in wood decay rates. Ecology 96(1):124-133.

van der Wal, A., et al.: 2016. Patterns of natural fungal community assembly during initial decay of coniferous and broadleaf tree logs. Ecosphere 7(7): 001393.

van der Wal, A., et al.: 2017. Fungal diversity and potential tree pathogens in decaying logs and stumps. Forest Ecology and Management 406:266-273. 10.3389/fmicb.2017.01028

10.1007/s11368-017-1701-9

10.1111/mec. 13680

10.1098/rsif.2015.1097

$10.1007 / \mathrm{s} 10530-017-1580-4$

10.1111/nph.15714

10.1093/femsec/fix031

10.1016/j.soilbio.2015.02.011

10.1016/j.myc.2016.02.001

10.1038/srep2775

10.1371/journal.pone.0153353

$10.1890 / 14-0242.1$

$10.1002 /$ ecs 2.1393

10.1016/j.foreco.2017.08.018 
van der Wal, A., et al.: 2017. Soil-wood interactions: Influence of decaying coniferous and broadleaf logs on composition of soil fungal communities. Fungal Ecology 30:132-134.

Vannette, R. L., et al.: 2016. Forest area and connectivity influence root-associated fungal communities in a fragmented landscape. Ecology 97(9):2374-2383.

Varenius, K., et al.: 2017. Retention of seed trees fails to lifeboat ectomycorrhizal fungal diversity in harvested Scots pine forests. FEMS Microbiology Ecology 93(9):fix105.

Vasutova, M., et al.: 2017. Distinct environmental variables drive the community composition of mycorrhizal and saprotrophic fungi at the alpine treeline ecotone. Fungal Ecology 27:116-124.

Vaz, A. B. M., et al.: 2017. Using Next-Generation Sequencing (NGS) to uncover diversity of wooddecaying fungi in neotropical Atlantic forests. Phytotaxa 295(1):1-21.

Veach, A. M., et al.: 2015. Woody plant encroachment, and its removal, impact bacterial and fungal communities across stream and terrestrial habitats in a tallgrass prairie ecosystem. FEMS Microbiology Ecology 91(10):fiv109.

Voriskova, J., et al.: 2014. Seasonal dynamics of fungal communities in a temperate oak forest soil. New Phytologist 201(1):269-278.

Walker, D. M., et al.: 2014. A metagenomics-based approach to the top-down effect on the detritivore food web: a salamanders influence on fungal communities within a deciduous forest. Ecology and Evolution 4(21): 4106-4116.

Wang, M. A., et al.: 2017. Influence of Peanut Cultivars and Environmental Conditions on the Diversity and Community Composition of Pod Rot Soil Fungi in China. Mycobiology 45(4):392-400.

Wang, W., et al.: 2016. Endophytic bacterial and fungal microbiota in sprouts, roots and stems of rice (Oryza sativa L.). Microbiological Research 188:1-8.

Waring, B. G., et al.: 2016. Scale-dependent variation in nitrogen cycling and soil fungal communities along gradients of forest composition and age in regenerating tropical dry forests. New Phytologist 209(2):845-854.

Wicaksono, C. Y., et al.: 2017. Contracting montane cloud forests: a case study of the Andean alder (Alnus acuminata) and associated fungi in the Yungas.

Biotropica 49(2):141-152.

Wilhelm, R. C., et al.: 2017. A metagenomic survey of forest soil microbial communities more than a decade after timber harvesting. Scientific Data 4:170092. 10.1016/j.funeco.2017.08.006

10.1002/ecy.1472

10.1093/femsec/fix105

10.1016/j.funeco.2016.08.010

10.11646/phytotaxa.295.1.1

10.1093/femsec/fiv109

10.1111/nph.12481

10.1002/ece3.1259

10.5941/MYCO.2017.45.4.392

10.1016/j.micres.2016.04.009

10.1111/nph.13654

10.1111/btp.12394

10.1038/sdata.2017.92 
Xu, L. H., et al.: 2012. Linking fungal communities in roots, rhizosphere, and soil to the health status of Pisum sativum. FEMS Microbiology Ecology 82(3):736745.

Yamamoto, S., et al.: 2014. Spatial Segregation and Aggregation of Ectomycorrhizal and Root-Endophytic Fungi in the Seedlings of Two Quercus Species. PLoS One 9(5):e96363.

Yang, T., et al.: 2016. Carbon constrains fungal endophyte assemblages along the timberline.

Environmental Microbiology 18(8):2455-2469.

Yang, T., et al.: 2016. Fungal assemblages in different habitats in an Erman's Birch forest.. Frontiers in Microbiology 7:1368.

Yang, T., et al.: 2017. Fungal community assemblages in a high elevation desert environment: Absence of dispersal limitation and edaphic effects in surface soil. Soil Biology \& Biochemistry 115:393-402.

Yang, T., et al.: 2017. Soil fungal diversity in natural grasslands of the Tibetan Plateau: associations with plant diversity and productivity. New Phytologist 215(2):756-765.

Yao, F., et al.: 2013. Substantial compositional turnover of fungal communities in an alpine ridge-tosnowbed gradient. Molecular Ecology 22(19):50405052.

Yao, F., et al.: 2017. Microbial Taxa Distribution Is Associated with Ecological Trophic Cascades along an Elevation Gradient. Frontiers in Microbiology 8:2071. Younginger, B. S. and Ballhorn, D. J.: 2017. Fungal endophyte communities in the temperate fern Polystichum munitum show early colonization and extensive temporal turnover. American Journal of Botany 104(8):1188-1194.

Zhang, J., et al.: 2018. Distinct large-scale biogeographic patterns of fungal communities in bulk soil and soybean rhizosphere in China. Science of the Total Environment 644:791-800.

Zhang, S. W., et al.: 2017. Relations among epiphytic microbial communities from soil, leaves and grapes of the grapevine. Frontiers in Life Science 10(1):73-83.

Zhang, T., et al.: 2015. Diversity and distribution of lichen-associated fungi in the Ny-Alesund Region (Svalbard, High Arctic) as revealed by 454 pyrosequencing. Scientific Reports 5:14850.

Zhang, T., et al.: 2016. Soil pH is a Key Determinant of Soil Fungal Community Composition in the NyÅlesund Region, Svalbard (High Arctic). Frontiers in Microbiology 7:227.

Zhang, W. W., et al.: 2017. Impacts of conversion from secondary forests to larch plantations on the 10.1111/j.1574-

6941.2012.01445.x

10.1371/journal.pone.0096363

$10.1111 / 1462-2920.13153$

10.3389/fmicb.2016.01368

10.1016/j.soilbio.2017.09.013

10.1111/nph.14606

10.1111/mec.12437

10.3389/fmicb.2017.02071

10.3732/ajb.1700149

10.1016/j.scitotenv.2018.07.016

$10.1080 / 21553769.2017 .1365776$

10.1038/srep14850

10.3389/fmicb.2016.00227

10.1016/j.apsoil.2016.11.019 
bioRxiv preprint doi: https://doi.org/10.1101/2020.04.24.060384; this version posted April 25, 2020. The copyright holder for this preprint (which was not certified by peer review) is the author/funder, who has granted bioRxiv a license to display the preprint in perpetuity. It is made available under aCC-BY-NC-ND 4.0 International license.

structure and function of microbial communities.

Applied Soil Ecology 111:73-83.

Zhang, Z. Q., et al.: 2017. Fungal communities in 10.1371/journal.pone.0187575 ancient peatlands developed from different periods in the Sanjiang Plain, China. PLoS One 12(12):e0187575. Zhou ,X. G., et al.: 2017. Conversion from long-term cultivated wheat field to Jerusalem artichoke plantation changed soil fungal communities. Scientific Reports 7:41502.

Zhou, J., et al.: 2016. Temperature mediates

10.1038/ncomms12083 continental-scale diversity of microbes in forest soils. Nature Communications 7:12083.

Zhou, X., et al.: 2017. Rhizospheric fungi and their link with the nitrogen-fixing Frankia harbored in host plant Hippophae rhamnoides L. Journal of Basic Microbiology 57(12):1055-1064.

Zifcakova, L., et al.: 2016. Microbial activity in forest soil reflects the changes in ecosystem properties between summer and winter. Environmental Microbiology 18(1):288-301.

Zimmerman, N. B. and Vitousek, P. M.: 2012. Fungal endophyte communities reflect environmental structuring across a Hawaiian landscape. Proceedings of the National Academy of Sciences of the United 10.1038/srep41502 States of America 109(32):13022-13027. 
bioRxiv preprint doi: https://doi.org/10.1101/2020.04.24.060384 this version posted April 25, 2020. The copyright holder for this preprin

(n)

made available under aCC-BY-NC-ND 4.0 international license.
a

\section{- \\ $\circ$}

\section{$\circ$}
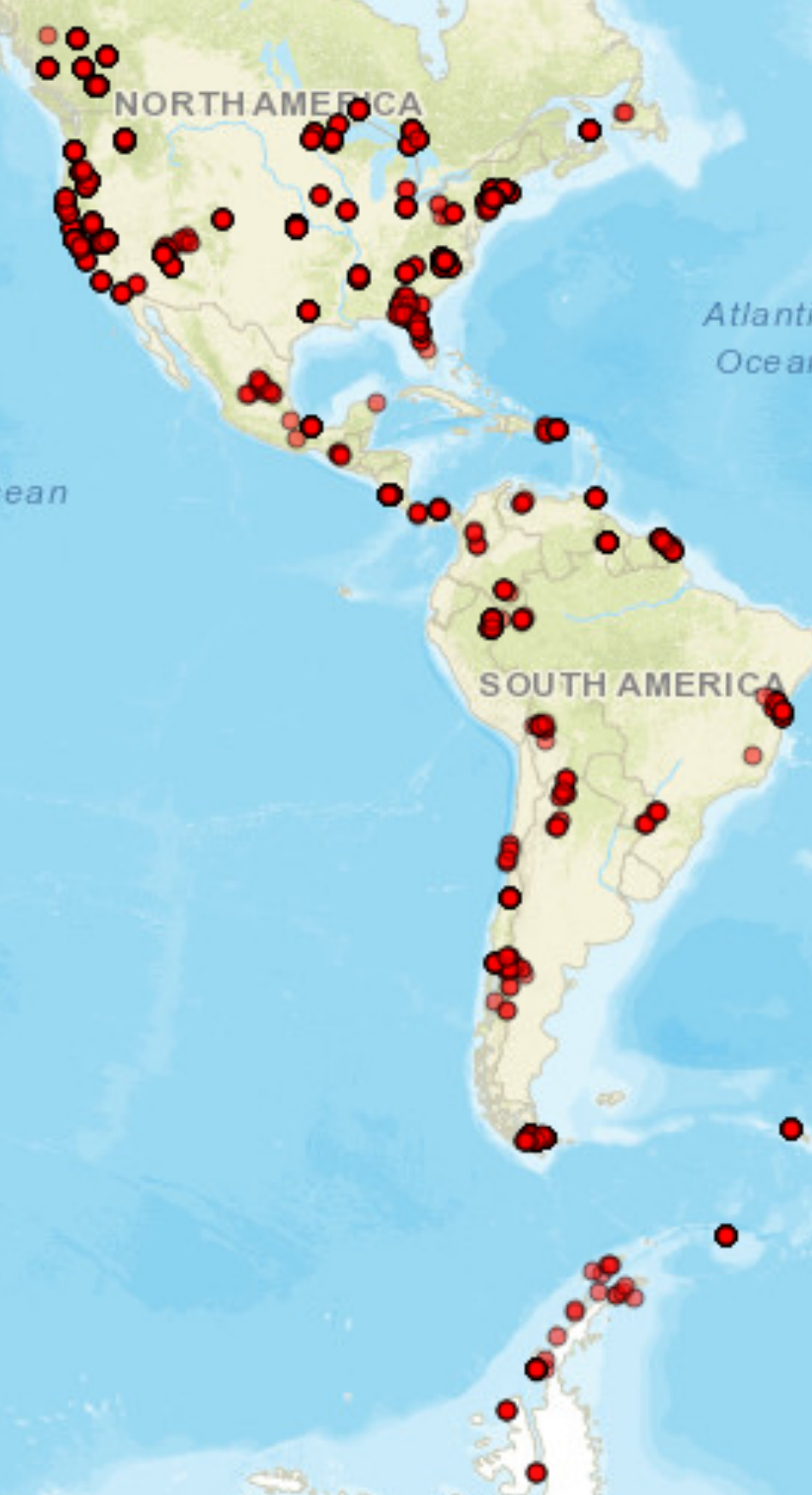

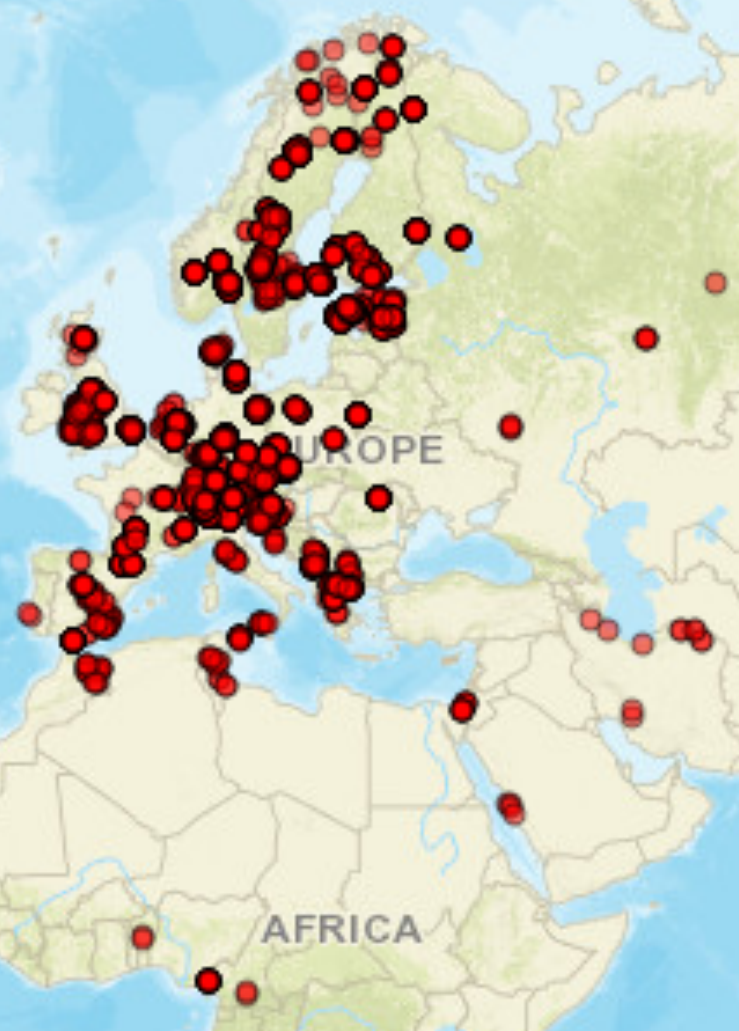

e

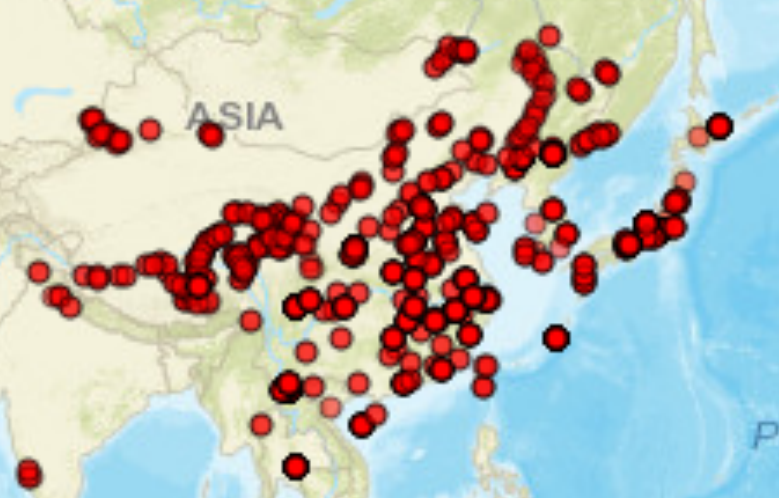

Pacific Ocean

- $\infty^{\circ}$

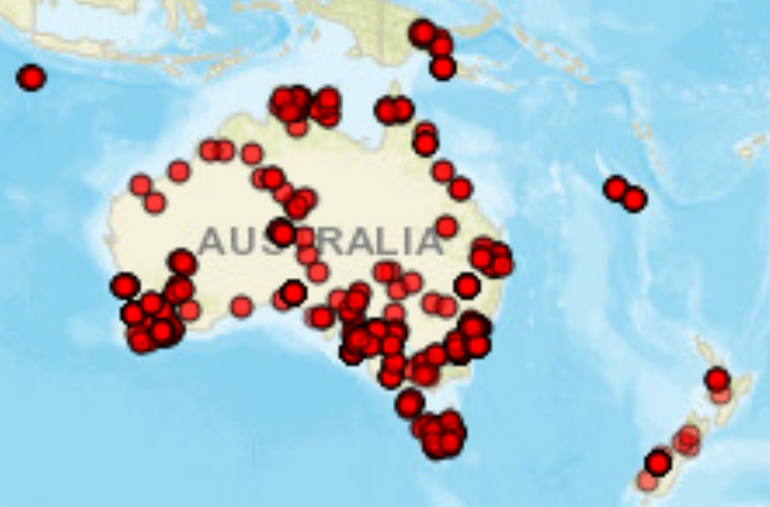

$8: 8$

Pacific Ocean

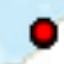


bioRxiv preprint doi: https://doi.org/10.1101/2020.04.24.060384; this versionposted April 252020 . The copyright holder for this preprint

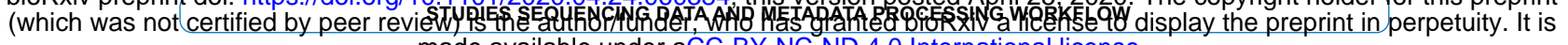
made available under aCC-BY-NC-ND 4.0 International license.

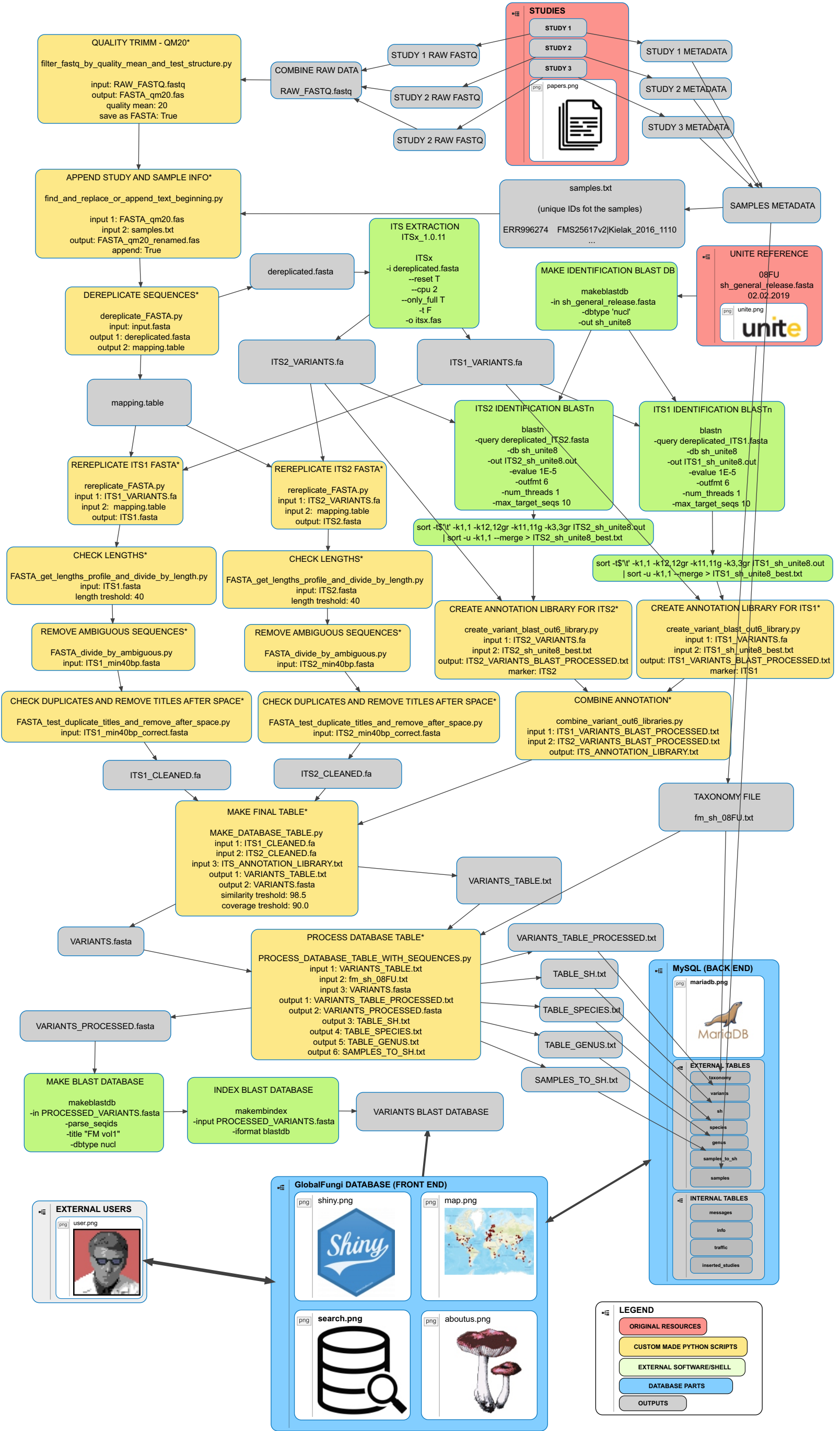


Database version vø.9.6; Release version 1.0; Taxonomy based on UNITE version 8.1(2.2.2019); Last update 27.3.2020; First release.

Actual number of samples in database 20009; Studies 207.
Number of ITS sequence variants 119566862; Number of ITS1 sequences 416291533; Number of ITS2 sequences 231278756.

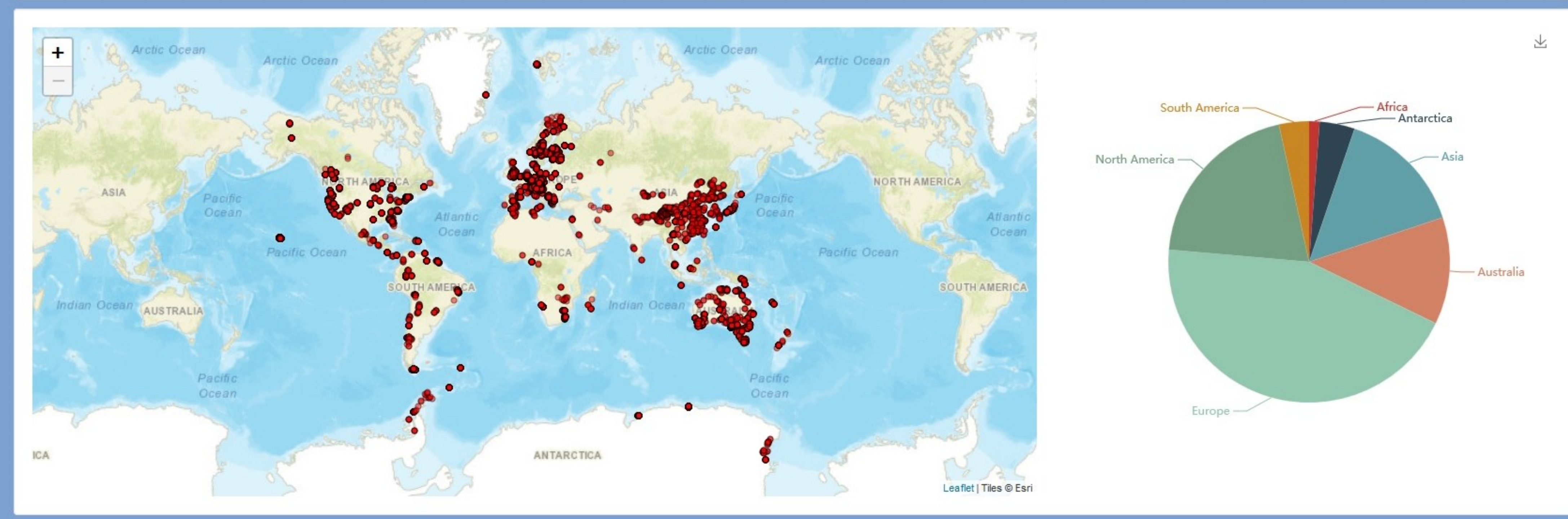

